\title{
The Non-Autonomous Chiral Model and the Ernst Equation of General Relativity in the Bidifferential Calculus Framework
}

\author{
Aristophanes DIMAKIS ${ }^{\dagger}$, Nils KANNING ${ }^{\ddagger}$ and Folkert MÜLLER-HOISSEN ${ }^{\S}$ \\ $\dagger$ Department of Financial and Management Engineering, University of the Aegean, \\ 41, Kountourioti Str., 82100 Chios, Greece \\ E-mail:dimakis@aegean.gr \\ $\ddagger$ Institute for Mathematics and Institute for Physics, Humboldt University, \\ Rudower Chaussee 25, 12489 Berlin, Germany \\ E-mail: kanning@mathematik.hu-berlin.de \\ $\S$ Max-Planck-Institute for Dynamics and Self-Organization, \\ Bunsenstrasse 10, 37073 Göttingen, Germany \\ E-mail: folkert.mueller-hoissen@ds.mpg.de
}

Received August 31, 2011, in final form December 16, 2011; Published online December 23, 2011 http://dx.doi.org/10.3842/SIGMA.2011.118

\begin{abstract}
The non-autonomous chiral model equation for an $m \times m$ matrix function on a two-dimensional space appears in particular in general relativity, where for $m=2$ a certain reduction of it determines stationary, axially symmetric solutions of Einstein's vacuum equations, and for $m=3$ solutions of the Einstein-Maxwell equations. Using a very simple and general result of the bidifferential calculus approach to integrable partial differential and difference equations, we generate a large class of exact solutions of this chiral model. The solutions are parametrized by a set of matrices, the size of which can be arbitrarily large. The matrices are subject to a Sylvester equation that has to be solved and generically admits a unique solution. By imposing the aforementioned reductions on the matrix data, we recover the Ernst potentials of multi-Kerr-NUT and multi-DemiańskiNewman metrics.
\end{abstract}

Key words: bidifferential calculus; chiral model; Ernst equation; Sylvester equation

2010 Mathematics Subject Classification: 37K10; 16E45

\section{Introduction}

The bidifferential calculus framework allows to elaborate solution generating methods for a wide class of nonlinear "integrable" partial differential or difference equations (PDDEs) to a considerable extent on a universal level, i.e. resolved from specific examples. It takes advantage of the simple rules underlying the calculus of differential forms (on a manifold), but allows for a generalization of the latter, which is partly inspired by noncommutative geometry. For a brief account of the basic structures and some results we refer to [1] (also see the references therein), but all what is needed for the present work is provided in Section 2. In this framework we explore the non-autonomous chiral model equation

$$
\left(\rho g_{z} g^{-1}\right)_{z}+\left(\rho g_{\rho} g^{-1}\right)_{\rho}=0
$$

for an $m \times m$ matrix $g$, where $\rho>0$ and $z$ are independent real variables, and a subscript indicates a corresponding partial derivative. It apparently first appeared, supplemented by certain reduction conditions (see Section 5), as the central part of the stationary axially symmetric Einstein 
$\operatorname{vacuum}(m=2)$ and Einstein-Maxwell $(m=3)$ equations (see in particular $[2,3,4,5,6,7,8])$. For $m>3$ this equation is met in higher-dimensional gravity, with a correspondingly enlarged number of Killing vector fields (see e.g. [9, 10, 11, 12, 13, 14, 15, 16, 17, 18, 19]). A version of the above equation also arises as the cylindrically symmetric case of the $(2+1)$-dimensional principal chiral model [20] and as a special case of the stationary Landau-Lifshitz equation for an isotropic two-dimensional ferromagnet [21].

The first construction of "multi-soliton" solutions of (1.1) has been carried out by Belinski and Zakharov [5, 6] (also see [7]) using the "dressing method" ${ }^{1}$. Here (1.1) is expressed as the integrability condition of a linear system, which depends on a (spectral) parameter and involves derivatives with respect to the latter. Another approach is based on a linear system that depends on a variable spectral parameter, i.e. a parameter that depends on the variables $\rho$ and $z$ [3]. In Appendix B we show that both linear systems arise from a universal linear system (see Section 2) in the bidifferential calculus framework (also see [31] for a relation between the two linear systems).

In the present work, we concentrate on a surprisingly simple general solution generating result in the bidifferential calculus framework, which has already been successfully applied in various other cases of integrable (soliton) equations [1,32, 33, 34] to generate multi-soliton families. In order to make it applicable to the non-autonomous chiral model, a slight generalization is required, however (see Section 3 and Appendix A). Section 4 then elaborates it for the $m \times m$ non-autonomous chiral model. We obtain solutions parametrized by four matrices. Two of them arise as solutions of an $n \times n$ matrix version of the quadratic equation for pole trajectories that first appeared in the solution generating method of Belinski and Zakharov [5, 6, 7]. It then remains to solve a Sylvester equation, where two more matrices enter, which are constant of size $m \times n$, respectively $n \times m$. Since $n$ can be arbitrarily large, we obtain an infinite family of solutions. The Sylvester equation is easily solved if the first two matrices are chosen diagonal, and in this case one recovers "multi-soliton" solutions. Additional solutions are obtained if the two $n \times n$ matrices are non-diagonal. In this case it is more difficult to solve the Sylvester equation, though a not very restrictive spectrum condition ensures the existence of a unique solution. Except for an example in Section 5, we will not elaborate this case further in this work.

Section 5 addresses reductions, in particular to the Ernst equation of general relativity. It turns out that the "multi-soliton" solutions of the stationary, axially symmetric Einstein vacuum and Einstein-Maxwell equations are indeed in the generated class of solutions of the nonautonomous chiral model. We thus obtain a new representation of these solutions. It has the property that the superposition of two (or more) "solitons" (e.g. black holes) simply corresponds to block-diagonal composition of the matrix data parametrizing the constituents. This puts a new perspective on an old result about one of the most important integrable equations in physics.

We would like to stress that the solutions of the non-autonomous chiral model and the Ernst equation(s), (re)derived in this work, originate from a universal result that also generates multi-soliton solutions of various other integrable equations in a non-iterative way. The crucial step is to find a "bidifferential calculus formulation" of the respective equation. This may be regarded as a generalization of the problem of formulating the equation as a reduction of the selfdual Yang-Mills equation. Indeed, in the case under consideration, it is of great help that an embedding of the non-autonomous chiral model in the $(m \times m)$ selfdual Yang-Mills equation is known [35, 36, 37, 38, 39, 40], and a bidifferential calculus formulation is then obtained from

\footnotetext{
${ }^{1}$ Further constructions of multi-soliton solutions, in the context of general relativity, were presented in particular by Alekseev [22, 23], Neugebauer [24], Kramer and Neugebauer [25, 26], Korotkin [27] (limiting cases of finite-gap solutions), Manko et al. [28, 29], and Masuda et al. [30] (using Hirota's method). Also see the references in [8, Section 34] for other solution generating techniques.
} 
that of the selfdual Yang-Mills equation [1], see Section 4. Once this is at hand, the remaining computations are rather straightforward.

Section 6 contains some concluding remarks.

\section{Preliminaries}

Basic definitions. A graded algebra is an associative algebra $\Omega$ over $\mathbb{C}$ with a direct sum decomposition $\Omega=\bigoplus_{r \geq 0} \Omega^{r}$ into a subalgebra $\mathcal{A}:=\Omega^{0}$ and $\mathcal{A}$-bimodules $\Omega^{r}$, such that $\Omega^{r} \Omega^{s} \subseteq$ $\Omega^{r+s}$. A bidifferential calculus (or bidifferential graded algebra) is a unital graded algebra $\Omega$ equipped with two (C-linear) graded derivations $\mathrm{d}, \overline{\mathrm{d}}: \Omega \rightarrow \Omega$ of degree one (hence $\mathrm{d} \Omega^{r} \subseteq \Omega^{r+1}$, $\left.\overline{\mathrm{d}} \Omega^{r} \subseteq \Omega^{r+1}\right)$, with the properties

$$
\mathrm{d}_{\kappa}^{2}=0 \quad \forall \kappa \in \mathbb{C}, \quad \text { where } \quad \mathrm{d}_{\kappa}:=\overline{\mathrm{d}}-\kappa \mathrm{d},
$$

and the graded Leibniz rule

$$
\mathrm{d}_{\kappa}\left(\chi \chi^{\prime}\right)=\left(\mathrm{d}_{\kappa} \chi\right) \chi^{\prime}+(-1)^{r} \chi \mathrm{d}_{\kappa} \chi^{\prime}
$$

for all $\chi \in \Omega^{r}$ and $\chi^{\prime} \in \Omega$. This means that $\mathrm{d}$ and $\overline{\mathrm{d}}$ both satisfy the graded Leibniz rule. In Section 3 we consider a more narrow class of graded algebras. A bidifferential calculus within this class is then specified in Section 4.

Dressing a bidifferential calculus. Let $(\Omega, \mathrm{d}, \overline{\mathrm{d}})$ be a bidifferential calculus. Replacing $\mathrm{d}_{\kappa}$ in (2.1) by

$$
\mathrm{D}_{\kappa}:=\overline{\mathrm{d}}-\mathbb{A}-\kappa \mathrm{d},
$$

with a 1 -form $\mathbb{A}$ (i.e. an element of $\Omega^{1}$ ), the resulting condition $\mathrm{D}_{\kappa}^{2}=0$ (for all $\kappa \in \mathbb{C}$ ) can be expressed as

$$
\mathrm{d} \mathbb{A}=0 \quad \text { and } \quad \overline{\mathrm{d}} \mathbb{A}-\mathbb{A} \mathbb{A}=0 .
$$

If these equations are equivalent to a PDDE or a system of PDDEs for a set of functions, we say we have a bidifferential calculus formulation for it. This requires that $\mathbb{A}$ depends on these functions and the derivations $\mathrm{d}, \overline{\mathrm{d}}$ involve differential or difference operators. There are several ways to reduce the two equations (2.2) to a single one. Here we only consider two of them.

1. We can solve the first of (2.2) by setting

$$
\mathbb{A}=\mathrm{d} \phi .
$$

This converts the second of $(2.2)$ into

$$
\overline{\mathrm{d}} \mathrm{d} \phi=\mathrm{d} \phi \mathrm{d} \phi .
$$

This equation is obviously invariant under $\phi \mapsto \alpha \phi \alpha^{-1}+\beta$ with an invertible $\alpha \in \mathcal{A}$ satisfying $\mathrm{d} \alpha=\overline{\mathrm{d}} \alpha=0$, and $\beta \in \mathcal{A}$ satisfying $\mathrm{d} \beta=0$.

2. Alternatively, the second of equations (2.2) can be solved by setting

$$
\mathbb{A}=(\overline{\mathrm{d}} g) g^{-1},
$$

and the first equation then reads

$$
\mathrm{d}\left((\overline{\mathrm{d}} g) g^{-1}\right)=0 .
$$


This equation has the (independent left and right handed, i.e. chiral) symmetry

$$
g \mapsto \alpha g \beta,
$$

where $\alpha \in \mathcal{A}$ is d-constant ${ }^{2}$ and $\beta \in \mathcal{A}$ is $\overline{\mathrm{d}}$-constant, and both have to be invertible. Since

$$
\overline{\mathrm{d}}\left[\left(\mathrm{d} g^{-1}\right) g\right]=-\overline{\mathrm{d}}\left(g^{-1} \mathrm{~d} g\right)=g^{-1}\left[(\mathrm{~d} \overline{\mathrm{d}} g) g^{-1}-(\overline{\mathrm{d}} g) \mathrm{d} g^{-1}\right] g=g^{-1} \mathrm{~d}\left[(\overline{\mathrm{d}} g) g^{-1}\right] g,
$$

$g$ solves (2.4) iff $g^{-1}$ solves (2.4) with d and $\overline{\mathrm{d}}$ exchanged. In our central example, the nonautonomous chiral model, $g \mapsto g^{-1}$ becomes a symmetry.

Linear system. The compatibility condition of the linear equation

$$
\overline{\mathrm{d}} X=(\mathrm{d} X) P+\mathbb{A} X
$$

is

$$
0=\overline{\mathrm{d}}^{2} X=(\mathrm{d} X)[(\mathrm{d} P) P-\overline{\mathrm{d}} P]+\left(\overline{\mathrm{d}} \mathbb{A}-\mathbb{A}^{2}\right) X-(\mathrm{d} \mathbb{A}) X P .
$$

If $P$ satisfies

$$
\overline{\mathrm{d}} P=(\mathrm{d} P) P,
$$

this reduces to

$$
\left(\overline{\mathrm{d}} \mathbb{A}-\mathbb{A}^{2}\right) X=(\mathrm{d} \mathbb{A}) X P .
$$

For the above choices of $\mathbb{A}$, this implies the respective PDDE. Hence (2.7) is the source of a corresponding Lax pair, also see Appendix B.

As a consequence of (2.8), $P$ solves (2.3) and, if $P$ is invertible, also (2.4).

Miura transformation. If a pair $(\phi, g)$ solves the Miura transformation equation

$$
(\overline{\mathrm{d}} g) g^{-1}=\mathrm{d} \phi
$$

(cf. [1]), it follows (as an integrability condition) that $\phi$ solves (2.3) and $g$ solves (2.4). We note that (2.10) is just the linear equation (2.7) if we identify $\mathbb{A}=\mathrm{d} \phi, X=g$ and set $P=0$. If we have chosen a bidifferential calculus and a reduction condition such that (2.3) becomes equivalent to some PDDE, this does not necessarily mean that also (2.4) is equivalent to some "ordinary" PDDE. But for the central example of this work, the non-autonomous chiral model, such a mismatch does not occur. In fact, in Section 3 we will actually present a solution generating method for $(2.10)$.

\section{A solution generating method}

Let $\bigwedge\left(\mathbb{C}^{N}\right)$ denote the exterior (Grassmann) algebra of the vector space $\mathbb{C}^{N}$ and $\operatorname{Mat}(m, n, \mathcal{B})$ the set of $m \times n$ matrices with entries in some unital algebra $\mathcal{B}$. We choose $\mathcal{A}$ as the algebra of all finite-dimensional matrices (with entries in $\mathcal{B}$ ), where the product of two matrices is defined to be zero if the sizes of the two matrices do not match, and assume that $\Omega=\mathcal{A} \otimes \bigwedge\left(\mathbb{C}^{N}\right)$ is supplied with the structure of a bidifferential calculus. In the following, $I=I_{m}$ and $\boldsymbol{I}=\boldsymbol{I}_{n}$ denote the $m \times m$, respectively $n \times n$, identity matrix.

\footnotetext{
${ }^{2}$ Although not evident, we need not require $\overline{\mathrm{d}} \alpha=0$ in addition.
} 
Proposition 3.1. Let $\boldsymbol{P}, \boldsymbol{R}, \boldsymbol{X} \in \operatorname{Mat}(n, n, \mathcal{B})$ be invertible solutions of

$$
\begin{aligned}
& \overline{\mathrm{d}} \boldsymbol{P}=(\mathrm{d} \boldsymbol{P}) \boldsymbol{P}, \quad \overline{\mathrm{d}} \boldsymbol{R}=\boldsymbol{R} \mathrm{d} \boldsymbol{R}, \\
& \overline{\mathrm{d}} \boldsymbol{X}=(\mathrm{d} \boldsymbol{X}) \boldsymbol{P}-(\mathrm{d} \boldsymbol{R}) \boldsymbol{X}, \quad \boldsymbol{X} \boldsymbol{P}-\boldsymbol{R} \boldsymbol{X}=\boldsymbol{V} \boldsymbol{U},
\end{aligned}
$$

with $\mathrm{d}$ - and $\overline{\mathrm{d}}$-constant $\boldsymbol{U} \in \operatorname{Mat}(m, n, \mathcal{B}), \boldsymbol{V} \in \operatorname{Mat}(n, m, \mathcal{B})$. Then

$$
\phi=\boldsymbol{U} \boldsymbol{X}^{-1} \boldsymbol{V}, \quad g=I+\boldsymbol{U}(\boldsymbol{R} \boldsymbol{X})^{-1} \boldsymbol{V}
$$

solve the Miura transformation equation (2.10), and thus (2.3), respectively (2.4).

Proof. Using the last three of (3.1) we obtain

$$
\begin{aligned}
\overline{\mathrm{d}}(\boldsymbol{R} \boldsymbol{X})^{-1} & =-\boldsymbol{X}^{-1}\left[\overline{\mathrm{d}} \boldsymbol{X} \boldsymbol{X}^{-1}+\boldsymbol{R}^{-1} \overline{\mathrm{d}} \boldsymbol{R}\right] \boldsymbol{R}^{-1}=-\boldsymbol{X}^{-1}(\mathrm{~d} \boldsymbol{X}) \boldsymbol{X}^{-1}(\boldsymbol{X} \boldsymbol{P})(\boldsymbol{R} \boldsymbol{X})^{-1} \\
& =\left(\mathrm{d} \boldsymbol{X}^{-1}\right)\left[\boldsymbol{I}+\boldsymbol{V} \boldsymbol{U}(\boldsymbol{R} \boldsymbol{X})^{-1}\right] .
\end{aligned}
$$

Multiplication by $\boldsymbol{U}$ from the left and by $\boldsymbol{V}$ from the right, and using $\overline{\mathrm{d}} I=0$, leads to

$$
\overline{\mathrm{d}} g=\boldsymbol{U}\left(\mathrm{d} \boldsymbol{X}^{-1}\right) \boldsymbol{V} g=(\mathrm{d} \phi) g .
$$

Hence $\phi$ and $g$ solve the Miura transformation equation (2.10). We did not use the first of (3.1), but it arises as an integrability condition: $0=\overline{\mathrm{d}}^{2} \boldsymbol{X}=(\mathrm{d} \boldsymbol{X})[(\mathrm{d} \boldsymbol{P}) \boldsymbol{P}-\overline{\mathrm{d}} \boldsymbol{P}]$.

Remark 3.1. The third of (3.1), which has the form of the linear equation (2.7), is almost a consequence of the fourth, which is a Sylvester equation. Indeed, as a consequence of the Sylvester equation we have

$$
\begin{aligned}
0 & =\overline{\mathrm{d}}(\boldsymbol{R} \boldsymbol{X}-\boldsymbol{X} \boldsymbol{P}+\boldsymbol{V} \boldsymbol{U})=(\overline{\mathrm{d}} \boldsymbol{R}) \boldsymbol{X}+\boldsymbol{R} \overline{\mathrm{d}} \boldsymbol{X}-(\overline{\mathrm{d}} \boldsymbol{X}) \boldsymbol{P}-\boldsymbol{X} \mathrm{d} \boldsymbol{P} \\
& =\boldsymbol{R}[\overline{\mathrm{d}} \boldsymbol{X}+(\mathrm{d} \boldsymbol{R}) \boldsymbol{X}]-[\overline{\mathrm{d}} \boldsymbol{X}+\boldsymbol{X} \mathrm{d} \boldsymbol{P}] \boldsymbol{P} \\
& =\boldsymbol{R}[\overline{\mathrm{d}} \boldsymbol{X}+(\mathrm{d} \boldsymbol{R}) \boldsymbol{X}-(\mathrm{d} \boldsymbol{X}) \boldsymbol{P}]-[\overline{\mathrm{d}} \boldsymbol{X}+(\mathrm{d} \boldsymbol{R}) \boldsymbol{X}-(\mathrm{d} \boldsymbol{X}) \boldsymbol{P}] \boldsymbol{P}+\mathrm{d}(\boldsymbol{R} \boldsymbol{X}-\boldsymbol{X} \boldsymbol{P}) \boldsymbol{P},
\end{aligned}
$$

where the last term vanishes. If $\boldsymbol{P}$ and $\boldsymbol{R}$ are sufficiently independent, this implies that the third of (3.1) is satisfied. In particular, this holds if $\mathcal{B}$ is the algebra of complex functions of some variables and if $\boldsymbol{P}$ and $\boldsymbol{R}$ have no eigenvalue in common.

Appendix A explains how Proposition 3.1 arises from a theorem that has been applied in previous work to generate soliton solutions of several integrable PDDEs.

\section{The non-autonomous chiral model}

The PDE defining the non-autonomous chiral model can be obtained as a reduction of the self-dual Yang-Mills (sdYM) equation (see e.g. [35, 36, 37, 38, 39, 40]). In an analogous way, a bidifferential calculus for the non-autonomous chiral model can be derived from a bidifferential calculus for the sdYM equation (also see [41]). In coordinates $\rho, z, \theta$, where $\rho>0$, it is given by

$$
\mathrm{d} f=-f_{z} \zeta_{1}+e^{\theta}\left(f_{\rho}-\rho^{-1} f_{\theta}\right) \zeta_{2}, \quad \overline{\mathrm{d}} f=e^{-\theta}\left(f_{\rho}+\rho^{-1} f_{\theta}\right) \zeta_{1}+f_{z} \zeta_{2} .
$$

Here e.g. $f_{z}$ denotes the partial derivative of a function $f$ (of the three coordinates) with respect to $z$, and $\zeta_{1}, \zeta_{2}$ is a basis of $\bigwedge^{1}\left(\mathbb{C}^{2}\right)$. d and $\overline{\mathrm{d}}$ extend to matrices of functions and moreover to $\Omega=\mathcal{A} \otimes \wedge\left(\mathbb{C}^{2}\right)$ with $\mathcal{A}=\operatorname{Mat}(m, m, \mathbb{C})$, treating $\zeta_{1}, \zeta_{2}$ as constants. The coordinate $\theta$ is needed to have the properties of a bidifferential calculus, but we are finally interested in equations for objects that do not depend on it. 
A (matrix-valued) function is d-constant ( $\overline{\mathrm{d}}$-constant) iff it is $z$-independent and only depends on the variables $\theta, \rho$ through the combination $\rho e^{\theta}$ (respectively $\rho e^{-\theta}$ ). It is $\mathrm{d}-$ and $\overline{\mathrm{d}}$-constant iff it is constant, i.e. independent of $z, \theta, \rho$.

For an $m \times m$ matrix-valued function $g$, (2.4) takes the form

$$
\left(\rho g_{z} g^{-1}\right)_{z}+\left(\rho g_{\rho} g^{-1}\right)_{\rho}-\left(g_{\rho} g^{-1}\right)_{\theta}+\left(g_{\theta} g^{-1}\right)_{\rho}-\rho^{-1}\left(g_{\theta} g^{-1}\right)_{\theta}=0 .
$$

Restricting $g$ by setting

$$
g=e^{c \theta} \tilde{g}
$$

with any constant $c$ and $\theta$-independent $\tilde{g}$, for the latter we obtain the non-autonomous chiral model equation ${ }^{3}$

$$
\left(\rho \tilde{g}_{z} \tilde{g}^{-1}\right)_{z}+\left(\rho \tilde{g}_{\rho} \tilde{g}^{-1}\right)_{\rho}=0 .
$$

In Section 4.1, we derive a family of exact solutions by application of Proposition 3.1. In Appendix B we recover two familiar linear systems (Lax pairs) for this equation.

Miura transformation. Evaluating (2.3) with

$$
\phi=e^{-\theta} \tilde{\phi},
$$

where $\tilde{\phi}$ is $\theta$-independent, we obtain

$$
\tilde{\phi}_{z z}+\tilde{\phi}_{\rho \rho}+\rho^{-1} \tilde{\phi}_{\rho}=\left[\tilde{\phi}_{\rho}+\rho^{-1} \tilde{\phi}, \tilde{\phi}_{z}\right]
$$

which is related to the non-autonomous chiral model by the Miura transformation

$$
\tilde{\phi}_{z}=-\tilde{g}_{\rho} \tilde{g}^{-1}-c \rho^{-1} I, \quad \tilde{g}_{z} \tilde{g}^{-1}=\tilde{\phi}_{\rho}+\rho^{-1} \tilde{\phi} .
$$

Symmetries. (4.2) is invariant under each of the following transformations, and thus, more generally, any combination of them.

(1) $\tilde{g} \mapsto \alpha \tilde{g} \beta$, with any invertible constant $m \times m$ matrices $\alpha$ and $\beta$ (cf. (2.5)).

(2) $\tilde{g} \mapsto \rho^{c} \tilde{g}$ with any constant $c$.

(3) $\tilde{g} \mapsto \tilde{g}^{-1}$ (also see $\left.(2.6)\right)$.

(4) $\tilde{g} \mapsto \tilde{g}^{\dagger}$, where ${ }^{\dagger}$ indicates Hermitian conjugation.

We note that $\tilde{g} \mapsto\left(\tilde{g}^{\dagger}\right)^{-1}$ is a fairly obvious symmetry. With its help, (4) follows immediately from (3).

\subsection{A family of exact solutions}

Let us first consider the equation $\overline{\mathrm{d}} \boldsymbol{P}=(\mathrm{d} \boldsymbol{P}) \boldsymbol{P}$, which is the first of (3.1). Using the above bidifferential calculus, it takes the form

$$
\boldsymbol{P}_{z} \boldsymbol{P}=-e^{-\theta}\left(\boldsymbol{P}_{\rho}+\rho^{-1} \boldsymbol{P}_{\theta}\right), \quad \boldsymbol{P}_{z}=e^{\theta}\left(\boldsymbol{P}_{\rho}-\rho^{-1} \boldsymbol{P}_{\theta}\right) \boldsymbol{P} .
$$

Writing

$$
\boldsymbol{P}=e^{-\theta} \tilde{\boldsymbol{P}},
$$

and assuming that $\tilde{\boldsymbol{P}}$ does not depend on $\theta$, this translates to

$$
\tilde{\boldsymbol{P}}_{\rho}-\rho^{-1} \tilde{\boldsymbol{P}}=-\tilde{\boldsymbol{P}}_{z} \tilde{\boldsymbol{P}}, \quad \tilde{\boldsymbol{P}}_{z}=\left(\tilde{\boldsymbol{P}}_{\rho}+\rho^{-1} \tilde{\boldsymbol{P}}\right) \tilde{\boldsymbol{P}} .
$$

The proof of the following result is provided in Appendix C.

\footnotetext{
${ }^{3}$ Changing the sign of the first term in the expression for $\mathrm{d} f$ in (4.1), we obtain a minus sign between the two terms on the left hand side of (4.2). This hyperbolic version of the chiral model shows up, in particular, in the reduction of the Einstein vacuum equations with two spacelike commuting Killing vector fields, describing gravitational plane waves [7]. Our further analysis can be adapted to this case.
} 
Lemma 4.1. The following holds.

(1) If $\tilde{\boldsymbol{P}}$ and $\boldsymbol{I}+\tilde{\boldsymbol{P}}^{2}$ are invertible, the system (4.4) implies

$$
\tilde{\boldsymbol{P}}^{2}-2 \rho^{-1}(z \boldsymbol{I}+\boldsymbol{B}) \tilde{\boldsymbol{P}}-\boldsymbol{I}=0
$$

with a constant matrix $\boldsymbol{B}$.

(2) Let $\boldsymbol{I}+\tilde{\boldsymbol{P}}^{2}$ be invertible and $\tilde{\boldsymbol{P}}_{\rho}, \tilde{\boldsymbol{P}}_{z}$ commute with $\tilde{\boldsymbol{P}}$. If $\tilde{\boldsymbol{P}}$ satisfies (4.5), then $\tilde{\boldsymbol{P}}$ solves (4.4).

Remark 4.1. If $\tilde{\boldsymbol{P}}$ is diagonal, then (4.5) becomes the set of quadratic equations (2.11) in [6] (or (1.67) in [7]), which determine the "pole trajectories" in the framework of Belinski and Zakharov. In our approach, there are more solutions since $\tilde{\boldsymbol{P}}$ need not be diagonal.

Remark 4.2. The conditions $\left[\tilde{\boldsymbol{P}}_{\rho}, \tilde{\boldsymbol{P}}\right]=\left[\tilde{\boldsymbol{P}}_{z}, \tilde{\boldsymbol{P}}\right]=0$ in part $(2)$ of the lemma are satisfied in particular if the spectrum $\operatorname{spec}(\boldsymbol{B})$ is simple, i.e. if the eigenvalues of $\boldsymbol{B}$ are all distinct, since then the solutions of (4.5) are functions of $\rho, z$ and the matrix $\boldsymbol{B}$ (and thus $\tilde{\boldsymbol{P}}_{\rho}$ and $\tilde{\boldsymbol{P}}_{z}$ commute with $\tilde{\boldsymbol{P}}$ ) [42]. But this would be unnecessarily restrictive, see Section 4.2.

Remark 4.3. Under the assumption that $\boldsymbol{I}+\tilde{\boldsymbol{P}}^{2}$ is invertible, $(4.4)$ implies $\left[\tilde{\boldsymbol{P}}_{\rho}, \tilde{\boldsymbol{P}}\right]=\left[\tilde{\boldsymbol{P}}_{z}, \tilde{\boldsymbol{P}}\right]=0$, also see (C.1). For the bidifferential calculus under consideration, $\overline{\mathrm{d}} \boldsymbol{P}=(\mathrm{d} \boldsymbol{P}) \boldsymbol{P}$ is therefore equivalent to $\overline{\mathrm{d}} \boldsymbol{P}=\boldsymbol{P} \mathrm{d} \boldsymbol{P}$. The latter is one of our equations for $\boldsymbol{R}$ in Proposition 3.1. Setting

$$
\boldsymbol{R}=e^{-\theta} \tilde{\boldsymbol{R}}
$$

with $\tilde{\boldsymbol{R}} \theta$-independent, invertible $\tilde{\boldsymbol{P}}$ and $\tilde{\boldsymbol{R}}$ both have to solve (4.5).

The third of (3.1) becomes

$$
\boldsymbol{X}_{\rho}+\rho^{-1} \boldsymbol{X}_{\theta}=-\boldsymbol{X}_{z} \tilde{\boldsymbol{P}}+\tilde{\boldsymbol{R}}_{z} \boldsymbol{X}, \quad \boldsymbol{X}_{z}=\left(\boldsymbol{X}_{\rho}-\rho^{-1} \boldsymbol{X}_{\theta}\right) \tilde{\boldsymbol{P}}-\left(\tilde{\boldsymbol{R}}_{\rho}+\rho^{-1} \tilde{\boldsymbol{R}}\right) \boldsymbol{X} .
$$

Assuming that $\boldsymbol{U}$ and $\boldsymbol{V}$ are $\theta$-independent, and recalling the $\theta$-dependence of $\phi$, the formula for $\phi$ in (3.2) requires $\boldsymbol{X}=e^{\theta} \tilde{\boldsymbol{X}}$ with $\theta$-independent $\tilde{\boldsymbol{X}}$. Hence

$$
\tilde{\boldsymbol{X}}_{\rho}+\rho^{-1} \tilde{\boldsymbol{X}}=-\tilde{\boldsymbol{X}}_{z} \tilde{\boldsymbol{P}}+\tilde{\boldsymbol{R}}_{z} \tilde{\boldsymbol{X}}, \quad \tilde{\boldsymbol{X}}_{z}=\left(\tilde{\boldsymbol{X}}_{\rho}-\rho^{-1} \tilde{\boldsymbol{X}}\right) \tilde{\boldsymbol{P}}-\left(\tilde{\boldsymbol{R}}_{\rho}+\rho^{-1} \tilde{\boldsymbol{R}}\right) \tilde{\boldsymbol{X}} .
$$

The last of (3.1) becomes the $\theta$-independent Sylvester equation

$$
\tilde{\boldsymbol{X}} \tilde{\boldsymbol{P}}-\tilde{\boldsymbol{R}} \tilde{\boldsymbol{X}}=\boldsymbol{V} \boldsymbol{U}
$$

Now Proposition 3.1 implies the following.

Proposition 4.1. Let $n \times n$ matrices $\tilde{\boldsymbol{P}}$ and $\tilde{\boldsymbol{R}}$ be solutions of (4.5) (with a matrix $\boldsymbol{B}$, respectively $\boldsymbol{B}^{\prime}$ ), with the properties that they commute with their derivatives w.r.t. $\rho$ and $z$, and that $\boldsymbol{I}+\tilde{\boldsymbol{P}}^{2}$ and $\boldsymbol{I}+\tilde{\boldsymbol{R}}^{2}$ are invertible. Furthermore, let $\operatorname{spec}(\tilde{\boldsymbol{P}}) \cap \operatorname{spec}(\tilde{\boldsymbol{R}})=\varnothing$ and $\tilde{\boldsymbol{X}}$ an invertible solution of the Sylvester equation (4.7) with constant $m \times n$, respectively $n \times m$, matrices $\boldsymbol{U}$ and $\boldsymbol{V}$. Then

$$
\tilde{g}=\left(I+\boldsymbol{U}(\tilde{\boldsymbol{R}} \tilde{\boldsymbol{X}})^{-1} \boldsymbol{V}\right) g_{0},
$$

with any constant invertible $m \times m$ matrix $g_{0}$, solves the non-autonomous chiral model equation (4.2).

\footnotetext{
${ }^{4}$ Here $g_{0}$ represents the freedom of chiral transformations.
} 
Proof. As a consequence of the spectrum condition, a solution $\tilde{\boldsymbol{X}}$ of the Sylvester equation (4.7) exists and is unique. The further assumptions for $\tilde{\boldsymbol{P}}$ and $\tilde{\boldsymbol{R}}$ are those of Lemma 4.1, part (2). Furthermore, (4.6) is a consequence of (4.7) if the spectrum condition holds (also see Remark 3.1). Now our assertion follows from Proposition 3.1 and the preceding calculations.

Remark 4.4. The determinant of (4.8) is obtained via Sylvester's theorem,

$$
\begin{aligned}
\operatorname{det}(\tilde{g}) & =\operatorname{det}\left(I+\boldsymbol{U}(\tilde{\boldsymbol{R}} \tilde{\boldsymbol{X}})^{-1} \boldsymbol{V}\right) \operatorname{det}\left(g_{0}\right)=\operatorname{det}\left(\boldsymbol{I}+\boldsymbol{V} \boldsymbol{U}(\tilde{\boldsymbol{R}} \tilde{\boldsymbol{X}})^{-1}\right) \operatorname{det}\left(g_{0}\right) \\
& =\operatorname{det}(\tilde{\boldsymbol{R}} \tilde{\boldsymbol{X}}+\boldsymbol{V} \boldsymbol{U}) \operatorname{det}(\tilde{\boldsymbol{R}} \tilde{\boldsymbol{X}})^{-1} \operatorname{det}\left(g_{0}\right)=\operatorname{det}(\tilde{\boldsymbol{X}} \tilde{\boldsymbol{P}}) \operatorname{det}(\tilde{\boldsymbol{R}} \tilde{\boldsymbol{X}})^{-1} \operatorname{det}\left(g_{0}\right) \\
& =\frac{\operatorname{det}(\tilde{\boldsymbol{P}})}{\operatorname{det}(\tilde{\boldsymbol{R}})} \operatorname{det}\left(g_{0}\right),
\end{aligned}
$$

where we used the Sylvester equation (4.7) and assumed that it has an invertible solution.

Remark 4.5. As an obvious consequence of (4.7), $\boldsymbol{U}$ and $\boldsymbol{V}$ enter $\tilde{g}$ given by (4.8) only modulo an arbitrary scalar factor different from zero. We also note that a transformation

$$
\tilde{\boldsymbol{P}} \mapsto \boldsymbol{T}_{1}^{-1} \tilde{\boldsymbol{P}} \boldsymbol{T}_{1}, \quad \tilde{\boldsymbol{R}} \mapsto \boldsymbol{T}_{2}^{-1} \tilde{R}_{2}, \quad \boldsymbol{U} \mapsto \boldsymbol{U}_{1}, \quad \boldsymbol{V} \mapsto \boldsymbol{T}_{2}^{-1} \boldsymbol{V}, \quad \tilde{\boldsymbol{X}} \mapsto \boldsymbol{T}_{2}^{-1} \tilde{\boldsymbol{X}} \boldsymbol{T}_{1},
$$

with constant invertible $n \times n$ matrices $\boldsymbol{T}_{1}, \boldsymbol{T}_{2}$, leaves (4.5), (4.6), (4.7) and (4.8) invariant. As a consequence, without restriction of generality, we can assume that the matrix $\boldsymbol{B}$ in (4.5), and the corresponding matrix related to $\tilde{\boldsymbol{R}}$, both have Jordan normal form.

Example 4.1. Let $\tilde{\boldsymbol{P}}$ and $\tilde{\boldsymbol{R}}$ be diagonal, i.e.

$$
\tilde{\boldsymbol{P}}=\left(p_{i} \delta_{i j}\right), \quad \tilde{\boldsymbol{R}}=\left(r_{i} \delta_{i j}\right) .
$$

If they have no eigenvalue in common, then (4.7) has a unique solution given by the Cauchy-like matrix

$$
\tilde{\boldsymbol{X}}_{i j}=\frac{(\boldsymbol{V U})_{i j}}{p_{j}-r_{i}} .
$$

It remains to solve (4.5) (choosing $\boldsymbol{B}$ diagonal), which yields

$$
p_{i}=\rho^{-1}\left(z+b_{i}+j_{i} \sqrt{\left(z+b_{i}\right)^{2}+\rho^{2}}\right), \quad r_{i}=\rho^{-1}\left(z+b_{i}^{\prime}+j_{i}^{\prime} \sqrt{\left(z+b_{i}^{\prime}\right)^{2}+\rho^{2}}\right)
$$

with constants $b_{i}, b_{i}^{\prime}$ and $j_{i}, j_{i}^{\prime} \in\{ \pm 1\}$. Since we assume that $\left\{p_{i}\right\} \cap\left\{r_{i}\right\}=\varnothing$, the assumptions of Proposition 4.1 are satisfied. It follows that, with the above data, (4.8) solves the nonautonomous chiral model equation.

The case where $\tilde{\boldsymbol{P}}$ or $\tilde{\boldsymbol{R}}$ is non-diagonal is exploited in the next subsection. But Example 4.1 will be sufficient to understand most of Section 5 .

\subsection{More about the family of solutions}

Introducing matrices $\boldsymbol{A}$ and $\boldsymbol{L}$ via

$$
\boldsymbol{A}=(z \boldsymbol{I}+\boldsymbol{B})^{2}+\rho^{2} \boldsymbol{I}, \quad \tilde{\boldsymbol{P}}=\rho^{-1}(\boldsymbol{L}+z \boldsymbol{I}+\boldsymbol{B}),
$$

(4.5) translates into

$$
\boldsymbol{L}^{2}=\boldsymbol{A}
$$


According to Remark 4.5, we can take $\boldsymbol{B}$ in Jordan normal form,

$$
\boldsymbol{B}=\text { block-diag }\left(\boldsymbol{B}_{n_{1}}, \ldots, \boldsymbol{B}_{n_{s}}\right) .
$$

Let us first consider the case where $\boldsymbol{B}$ is a single $r \times r$ Jordan block,

$$
\boldsymbol{B}_{r}=b \boldsymbol{I}_{r}+\boldsymbol{N}_{r}, \quad \boldsymbol{N}_{r}=\left(\begin{array}{ccccc}
0 & 1 & 0 & \cdots & 0 \\
0 & 0 & 1 & \ddots & \vdots \\
\vdots & & \ddots & \ddots & \vdots \\
\vdots & & & \ddots & 1 \\
0 & \cdots & \cdots & \cdots & 0
\end{array}\right) .
$$

Then we have

$$
\boldsymbol{A}=\mathfrak{r}^{2}\left(\boldsymbol{I}_{r}+\boldsymbol{M}_{r}\right),
$$

where

$$
\boldsymbol{M}_{r}=\mathfrak{r}^{-2}\left[2(z+b) \boldsymbol{N}_{r}+\boldsymbol{N}_{r}^{2}\right], \quad \mathfrak{r}= \pm \sqrt{(z+b)^{2}+\rho^{2}},
$$

and thus

$$
\boldsymbol{L}=\mathfrak{r}\left(\boldsymbol{I}_{r}+\boldsymbol{M}_{r}\right)^{1 / 2}=\mathfrak{r} \sum_{k=0}^{r-1}\left(\begin{array}{c}
1 / 2 \\
k
\end{array}\right) \boldsymbol{M}_{r}^{k},
$$

by use of the generalized binomial expansion formula, noting that $\boldsymbol{M}_{r}^{r}=0$ as a consequence of $\boldsymbol{N}_{r}^{r}=0$. Hence we obtain the following solution of (4.5),

$$
\tilde{\boldsymbol{P}}_{r}=\rho^{-1}\left(z \boldsymbol{I}_{r}+\boldsymbol{B}_{r}+\mathfrak{r} \sum_{k=0}^{r-1}\left(\begin{array}{c}
1 / 2 \\
k
\end{array}\right) \boldsymbol{M}_{r}^{k}\right),
$$

which is an upper triangular Toeplitz matrix. In particular, we have

$$
\begin{aligned}
& \tilde{\boldsymbol{P}}_{1}=\rho^{-1}[z+b+\mathfrak{r}], \\
& \tilde{\boldsymbol{P}}_{2}=\rho^{-1}[z+b+\mathfrak{r}]\left(\begin{array}{cc}
1 & \mathfrak{r}^{-1} \\
0 & 1
\end{array}\right), \\
& \tilde{\boldsymbol{P}}_{3}=\rho^{-1}[z+b+\mathfrak{r}]\left(\begin{array}{ccc}
1 & \mathfrak{r}^{-1} & \frac{1}{2} \rho^{2} \mathfrak{r}^{-3}(z+b+\mathfrak{r})^{-1} \\
0 & 1 & \mathfrak{r}^{-1} \\
0 & 0 & 1
\end{array}\right), \\
& \tilde{\boldsymbol{P}}_{4}=\rho^{-1}[z+b+\mathfrak{r}]\left(\begin{array}{cccc}
1 & \mathfrak{r}^{-1} & \frac{1}{2} \rho^{2} \mathfrak{r}^{-3}(z+b+\mathfrak{r})^{-1} & -\frac{1}{2}(z+b) \rho^{2} \mathfrak{r}^{-5}(z+b+\mathfrak{r})^{-1} \\
0 & 1 & \mathfrak{r}^{-1} & \frac{1}{2} \rho^{2} \mathfrak{r}^{-3}(z+b+\mathfrak{r})^{-1} \\
0 & 0 & 1 & \mathfrak{r}^{-1} \\
0 & 0 & 0 & 1
\end{array}\right) .
\end{aligned}
$$

These matrices are obviously nested and, from one to the next, only the entry in the right upper corner is new.

For the above Jordan normal form of $\boldsymbol{B}$, solutions of (4.5) are now given by ${ }^{5}$

$$
\tilde{\boldsymbol{P}}=\operatorname{block}-\operatorname{diag}\left(\tilde{\boldsymbol{P}}_{n_{1}}, \ldots, \tilde{\boldsymbol{P}}_{n_{s}}\right),
$$

\footnotetext{
${ }^{5}$ For $\tilde{\boldsymbol{P}}$ with simple spectrum, every solution of (4.5) has this form. Otherwise, i.e. when there are two Jordan blocks with the same eigenvalue, (4.5) has additional solutions, see [42]. But they are further constrained by (4.4). We will not consider such solutions in this work.
} 
where the blocks typically involve different constants replacing $b$, i.e. different eigenvalues of $\boldsymbol{B}$. Since $\tilde{\boldsymbol{P}}_{\rho}$ and $\tilde{\boldsymbol{P}}_{z}$ obviously commute with $\tilde{\boldsymbol{P}}$, and since $\boldsymbol{I}+\tilde{\boldsymbol{P}}^{2}$ is invertible ${ }^{6}$ for $\rho>0$, Lemma 4.1 , part (2), ensures that $\tilde{\boldsymbol{P}}$ solves (4.4). If $\tilde{\boldsymbol{P}}$ has the above form, and $\tilde{\boldsymbol{R}}$ a similar form, and if $\tilde{\boldsymbol{P}}$ and $\tilde{\boldsymbol{R}}$ have disjoint spectra, it remains to solve the Sylvester equation ${ }^{7}$ (4.7) in order that (4.8) yields solutions of the non-autonomous chiral model equation. This leads to a plethora of exact solutions. We postpone an example to Section 5 , where additional conditions considerably reduce the freedom we have here, see Example 5.2.

\section{Reductions of the non-autonomous chiral model to Ernst equations}

According to Section 4, a particular involutive symmetry of the non-autonomous chiral model (4.2) is given by $\tilde{g} \mapsto \gamma\left(\tilde{g}^{\dagger}\right)^{-1} \gamma$, where $\gamma$ is a constant matrix with

$$
\gamma^{\dagger}=\gamma, \quad \gamma^{2}=I
$$

(4.2) therefore admits the generalized unitarity reduction $\tilde{g}^{\dagger} \gamma \tilde{g}=\gamma$, which means that $\tilde{g}$ belongs to the unitary group $U(m ; \gamma){ }^{8}$ Another reduction, associated with an involutive symmetry, is $\tilde{g}^{\dagger}=\tilde{g}$. Imposing both reductions simultaneously, amounts to setting

$$
\tilde{g}^{\dagger}=\tilde{g}, \quad(\gamma \tilde{g})^{2}=I .
$$

Writing

$$
\tilde{g}=\gamma(I-2 \mathcal{P})
$$

translates these conditions into

$$
\gamma \mathcal{P}^{\dagger} \gamma=\mathcal{P}, \quad \mathcal{P}^{2}=\mathcal{P}
$$

In particular, $\mathcal{P}$ is a projector. If we require in addition that $\operatorname{rank}(\mathcal{P})=1$, which for a projector is equivalent to $\operatorname{tr}(\mathcal{P})=1$ [43, Fact 5.8.1], the following parametrization of $\tilde{g}$ can be achieved (also see e.g. [44, 45, 46, 47]),

$$
\tilde{g}=\gamma-2 \frac{v v^{\dagger}}{v^{\dagger} \gamma v}
$$

where $v$ is an $m$-component vector with $v^{\dagger} \gamma v \neq 0$. This parametrization is invariant under $v \mapsto c v$ with a nowhere vanishing function $c$, so that the first component of $v$ can be set to 1 in the generic case where it is different from zero. If $\gamma$ has signature $m-1,(5.2)$ is a parametrization of the symmetric space $S U(m-1,1) / S(U(m-1) \times U(1))[44,45,46]$. The condition $\operatorname{tr}(\mathcal{P})=1$ corresponds to

$$
\operatorname{tr}(\gamma \tilde{g})=m-2 .
$$

We also note that $\operatorname{det}(\tilde{g})=-\operatorname{det}(\gamma)$. The following result, which we prove in Appendix $\mathrm{C}$, shows how the reduction conditions (5.1) and (5.3) can be implemented on the family of solutions of the non-autonomous chiral model obtained via Proposition 4.1.

\footnotetext{
${ }^{6}$ Note that $I_{r}+\tilde{\boldsymbol{P}}_{r}^{2}=\left(1+\rho^{-2}(z+b+\mathfrak{r})^{2}\right) I+\sum_{k=1}^{r-1} g_{k} \boldsymbol{N}_{r}^{k}$ with functions $g_{k}$.

${ }^{7}$ Under the stated conditions the Sylvester equation possesses a unique solution and a vast literature exists to express it.

${ }^{8}$ If $\gamma$ has $p$ positive and $q$ negative eigenvalues, this is commonly denoted $U(p, q)$.
} 
Proposition 5.1. Let $\tilde{\boldsymbol{X}}$ solve the Sylvester equation (4.7), where $\tilde{\boldsymbol{P}}, \tilde{\boldsymbol{R}}, \boldsymbol{U}, \boldsymbol{V}$, satisfy ${ }^{9}$

$$
(\boldsymbol{\Gamma} \tilde{\boldsymbol{P}})^{2}=-\boldsymbol{I}, \quad(\boldsymbol{\Gamma} \tilde{\boldsymbol{R}})^{2}=-\boldsymbol{I}, \quad g_{0} \gamma \boldsymbol{U}=\boldsymbol{U} \boldsymbol{\Gamma}, \quad \boldsymbol{\Gamma} \boldsymbol{V}=\boldsymbol{V} g_{0} \gamma,
$$

with an $n \times n$ matrix $\boldsymbol{\Gamma}$ and a constant $m \times m$ matrix $g_{0}$ satisfying

$$
\boldsymbol{\Gamma}^{2}=\boldsymbol{I}, \quad\left(g_{0} \gamma\right)^{2}=I .
$$

Furthermore, let $\operatorname{spec}(\tilde{\boldsymbol{P}}) \cap \operatorname{spec}(\tilde{\boldsymbol{R}})=\varnothing$.

(1) $\tilde{g}$ given by (4.8) satisfies

$$
(\gamma \tilde{g})^{2}=I \quad \text { and } \quad \operatorname{tr}(\gamma \tilde{g})=\operatorname{tr}\left(\gamma g_{0}\right)-2 \operatorname{tr}(\boldsymbol{\Gamma}) .
$$

(2) If moreover the relations

$$
\tilde{\boldsymbol{R}}^{\dagger}=\boldsymbol{\Gamma} \tilde{\boldsymbol{P}} \boldsymbol{\Gamma}, \quad \boldsymbol{U}^{\dagger}=\boldsymbol{V} g_{0}, \quad g_{0}^{\dagger}=g_{0}, \quad \boldsymbol{\Gamma}^{\dagger}=\boldsymbol{\Gamma}
$$

hold, then $\tilde{g}$ given by (4.8) is Hermitian.

Remark 5.1. Let the matrix data $\left(\tilde{\boldsymbol{P}}_{i}, \tilde{\boldsymbol{R}}_{i}, \boldsymbol{U}_{i}, \boldsymbol{V}_{i}, \boldsymbol{\Gamma}_{i}\right)$ satisfy $\boldsymbol{\Gamma}_{i}^{2}=\boldsymbol{I}_{n_{i}}$ and

$$
\left(\boldsymbol{\Gamma}_{i} \tilde{\boldsymbol{P}}_{i}\right)^{2}=-\boldsymbol{I}_{n_{i}}, \quad\left(\boldsymbol{\Gamma}_{i} \tilde{\boldsymbol{R}}_{i}\right)^{2}=-\boldsymbol{I}_{n_{i}}, \quad g_{0} \gamma \boldsymbol{U}_{i}=\boldsymbol{U}_{i} \boldsymbol{\Gamma}_{i}, \quad \boldsymbol{\Gamma}_{i} \boldsymbol{V}_{i}=\boldsymbol{V}_{i} g_{0} \gamma
$$

Set $\tilde{\boldsymbol{P}}=\operatorname{block}-\operatorname{diag}\left(\tilde{\boldsymbol{P}}_{1}, \ldots, \tilde{\boldsymbol{P}}_{N}\right), \tilde{\boldsymbol{R}}=\operatorname{block}-\operatorname{diag}\left(\tilde{\boldsymbol{R}}_{1}, \ldots, \tilde{\boldsymbol{R}}_{N}\right), \boldsymbol{\Gamma}=\operatorname{block}-\operatorname{diag}\left(\boldsymbol{\Gamma}_{1}, \ldots, \boldsymbol{\Gamma}_{N}\right)$, and

$$
\boldsymbol{U}=\left(\boldsymbol{U}_{1}, \ldots, \boldsymbol{U}_{N}\right), \quad \boldsymbol{V}=\left(\begin{array}{c}
\boldsymbol{V}_{1} \\
\vdots \\
\boldsymbol{V}_{N}
\end{array}\right)
$$

Then we have $\Gamma^{2}=\boldsymbol{I}$ and $(5.4)$ holds. If $\operatorname{spec}(\tilde{\boldsymbol{P}}) \cap \operatorname{spec}(\tilde{\boldsymbol{R}})=\varnothing$, the corresponding Sylvester equation has a unique solution $\tilde{\boldsymbol{X}}$. According to part (1) of Proposition 5.1, $\tilde{g}$ given by (4.8) satisfies the reduction conditions (5.6). This is a way to superpose solutions from the class obtained in Section 3, preserving the constraints (5.4). We simply block-diagonally compose the matrix data associated with the constituents. In an obvious way, this method can be extended to part (2) of Proposition 5.1.

Remark 5.2. Let $n=2 N$ and

$$
\tilde{\boldsymbol{P}}=\left(\begin{array}{cc}
\check{\boldsymbol{P}} & 0 \\
0 & -\check{\boldsymbol{P}}^{-1}
\end{array}\right), \quad \tilde{\boldsymbol{R}}=\left(\begin{array}{cc}
\check{\boldsymbol{R}} & 0 \\
0 & -\check{\boldsymbol{R}}^{-1}
\end{array}\right), \quad \boldsymbol{\Gamma}=\left(\begin{array}{cc}
0 & \mathrm{i} \boldsymbol{I}_{N} \\
-\mathrm{i} \boldsymbol{I}_{N} & 0
\end{array}\right),
$$

where $\check{\boldsymbol{P}}$ and $\check{\boldsymbol{R}}$ are invertible block-diagonal $N \times N$ matrices, composed of blocks of the form (4.10). Then we have $(\boldsymbol{\Gamma} \tilde{\boldsymbol{P}})^{2}=-\boldsymbol{I}_{n}$ and $(\boldsymbol{\Gamma} \tilde{\boldsymbol{R}})^{2}=-\boldsymbol{I}$. Choosing $\gamma$ and $g_{0}$ such that $\left(g_{0} \gamma\right)^{2}=I$, the conditions $g_{0} \gamma \boldsymbol{U}=\boldsymbol{U} \boldsymbol{\Gamma}$ and $\boldsymbol{\Gamma} \boldsymbol{V}=\boldsymbol{V} g_{0} \gamma$ are solved by

$$
\boldsymbol{U}=\left(\begin{array}{ll}
\check{\boldsymbol{U}} & \mathrm{i} g_{0} \gamma \check{\boldsymbol{U}}
\end{array}\right), \quad \boldsymbol{V}=\left(\begin{array}{c}
\check{\boldsymbol{V}} \\
-\mathrm{i} \check{\boldsymbol{V}} g_{0} \gamma
\end{array}\right),
$$

where $\check{\boldsymbol{U}}$ and $\check{\boldsymbol{V}}$ are arbitrary constant $m \times N$, respectively $N \times m$ matrices. Writing

$$
\tilde{\boldsymbol{X}}=\left(\begin{array}{cc}
\check{\boldsymbol{X}} & \check{\boldsymbol{R}}^{-1} \check{\boldsymbol{Z}} \check{\boldsymbol{P}} \\
\check{Z} & \check{\boldsymbol{R}} \check{\boldsymbol{X}} \check{\boldsymbol{P}}
\end{array}\right)
$$

\footnotetext{
${ }^{9}$ These conditions are motivated by the structure of $\tilde{\boldsymbol{P}}, \tilde{\boldsymbol{R}}, \boldsymbol{U}, \boldsymbol{V}$ found in Example 5.1.
} 
reduces the $2 N \times 2 N$ Sylvester equation (4.7) to the two $N \times N$ Sylvester equations

$$
\check{\boldsymbol{X}} \check{\boldsymbol{P}}-\check{\boldsymbol{R}} \check{\boldsymbol{X}}=\check{\boldsymbol{V}} \check{\boldsymbol{U}}, \quad \check{\boldsymbol{Z}} \check{\boldsymbol{P}}+\check{\boldsymbol{R}}^{-1} \check{\boldsymbol{Z}}=-\mathrm{i} \check{\boldsymbol{V}} g_{0} \gamma \check{\boldsymbol{U}}
$$

If $\check{\boldsymbol{X}}$ and $\check{\boldsymbol{Z}}$ are invertibel, then $\tilde{\boldsymbol{R}} \tilde{\boldsymbol{X}}$ is invertible ${ }^{10}$. Proposition 5.1, part (1), implies that (4.8) with the above matrix data satisfies $(\gamma \tilde{g})^{2}=I$ and $\operatorname{tr}(\gamma \tilde{g})=\operatorname{tr}\left(\gamma g_{0}\right)$. With a suitable choice of $\gamma$ and $g_{0}$ we can achieve that (5.3) holds. To fulfil the remaining Hermiticity condition, one possibility is via part (2) of Proposition 5.1. See also Examples 5.2 and 5.7. Such solutions can be superposed in the way described in Remark 5.1.

In the special case where $\check{\boldsymbol{R}}=r \boldsymbol{I}_{N}$, the solutions of the Sylvester equations (5.8) are $\check{\boldsymbol{X}}=$ $\check{\boldsymbol{V}} \check{\boldsymbol{U}}\left(\check{\boldsymbol{P}}-r \boldsymbol{I}_{N}\right)^{-1}$ and $\check{\boldsymbol{Z}}=-\mathrm{i} \check{\boldsymbol{V}} g_{0} \gamma \check{\boldsymbol{U}}\left(\check{\boldsymbol{P}}+r^{-1} \boldsymbol{I}_{N}\right)^{-1}$. These expressions are not invertible if $N>m$, so in this particular case our solution formula only works for $N \leq m$ (also see Example 5.2).

Remark 5.3. The following observation in particular underlies the Harrison transformation $[48,49]$ which we consider in Example 5.5 below. Let $H$ be an $m \times m$-matrix that satisfies

$$
H^{\dagger} \gamma H=\gamma
$$

If $\tilde{g}$ satisfies

$$
(\gamma \tilde{g})^{2}=I, \quad \tilde{g}^{\dagger}=\tilde{g},
$$

then also

$$
\tilde{g}^{\prime}=H \tilde{g} H^{\dagger},
$$

and we have $\operatorname{tr}\left(\gamma \tilde{g}^{\prime}\right)=\operatorname{tr}(\gamma \tilde{g})$. If $\tilde{g}$ has the form (4.8) with Hermitian $g_{0}$ and $\left(\gamma g_{0}\right)^{2}=I$, and if $H$ also satisfies

$$
H g_{0} H^{\dagger}=g_{0},
$$

then the effect of the transformation $\tilde{g} \mapsto \tilde{g}^{\prime}$ amounts to the replacement

$$
\boldsymbol{U} \mapsto \boldsymbol{U}^{\prime}=H \boldsymbol{U}, \quad \boldsymbol{V} \mapsto \boldsymbol{V}^{\prime}=\boldsymbol{V} H^{-1},
$$

which leaves the Sylvester equation (4.7) invariant.

\subsection{Solutions of the Ernst equation of general relativity}

We choose $m=2$ and

$$
\gamma=\left(\begin{array}{cc}
0 & \mathrm{i} \\
-\mathrm{i} & 0
\end{array}\right)
$$

and write

$$
v=\left(\begin{array}{c}
1 \\
\mathrm{i} \mathcal{E}
\end{array}\right)
$$

with a complex function $\mathcal{E}$ and its complex conjugate $\overline{\mathcal{E}}$. Then (5.2) takes the form

$$
\tilde{g}=\frac{2}{\mathcal{E}+\overline{\mathcal{E}}}\left(\begin{array}{cc}
1 & \frac{\mathrm{i}}{2}(\mathcal{E}-\overline{\mathcal{E}}) \\
\frac{i}{2}(\mathcal{E}-\overline{\mathcal{E}}) & \overline{\mathcal{E}} \mathcal{E}
\end{array}\right)
$$

\footnotetext{
${ }^{10}(\tilde{\boldsymbol{R}} \tilde{\boldsymbol{X}})^{-1}$ can be computed as a $2 \times 2$ block matrix. The problem of evaluating the original expression for $\tilde{g}$ that involves $2 N \times 2 N$ matrices then reduces to that of evaluating only $N \times N$ matrix expressions.
} 
so that

$$
\mathcal{E}=\frac{1-\mathrm{i} \tilde{g}_{21}}{\tilde{g}_{11}}
$$

(4.2) now becomes the Ernst equation

$$
(\operatorname{Re} \mathcal{E})\left(\partial_{\rho}^{2}+\rho^{-1} \partial_{\rho}+\partial_{z}^{2}\right) \mathcal{E}=\left(\mathcal{E}_{\rho}\right)^{2}+\left(\mathcal{E}_{z}\right)^{2}
$$

where e.g. $\partial_{\rho}$ denotes the partial derivative with respect to $\rho$. This equation determines solutions of the stationary axially symmetric Einstein vacuum equations. The following statements are easily verified.

1. Excluding $\tilde{g}= \pm \gamma$, the reduction conditions (5.1) are equivalent to

$$
\tilde{g} \text { real, } \quad \operatorname{det}(\tilde{g})=1, \quad \tilde{g}_{12}=\tilde{g}_{21} \text {. }
$$

(5.3) is then automatically satisfied.

2. For real $\tilde{g}$, the second of the reduction conditions (5.1) implies the first. As a consequence, Proposition 5.1, part (1), already generates solutions of the Ernst equation.

We will use these observations in the following examples.

Example 5.1 (Kerr-NUT). For the solution of the non-autonomous chiral model given in Example 4.1 with $n=2$, we have (also see Remark 4.4)

$$
\operatorname{det}(\tilde{g})=\frac{p_{1} p_{2}}{r_{1} r_{2}} \operatorname{det}\left(g_{0}\right)
$$

with $p_{i}, r_{i}$ given by (4.9). Choosing

$$
g_{0}=I_{2}
$$

so that $\operatorname{det}\left(g_{0}\right)=1$, the second of the reduction conditions (5.11) is solved by setting

$$
p_{2}=-\frac{1}{p_{1}}, \quad r_{2}=-\frac{1}{r_{1}}
$$

noting that $-1 / p_{i}$ is given by the expression for $p_{i}$ with $j_{i}$ exchanged by $-j_{i}$. We shall write $p, r$ instead of $p_{1}, r_{1}$. With $\boldsymbol{U}=\left(u_{i j}\right), \boldsymbol{V}=\left(v_{i j}\right)$, the remaining constraint $\tilde{g}_{12}=\tilde{g}_{21}$ is solved by ${ }^{11}$

$$
u_{22}=-u_{11} u_{12} / u_{21}, \quad v_{22}=-v_{11} v_{21} / v_{12} .
$$

In the following we assume that $u_{11}$ and $v_{11}$ are different from zero and write

$$
u_{21}=u u_{11}, \quad v_{12}=v v_{11}
$$

Then $u_{11}, u_{12}, v_{11}, v_{21}$ drop out of $\tilde{g}$. Without restriction of generality, we can therefore choose them as $u_{11}=1, u_{12}=-u, v_{11}=1$ and $v_{21}=-v$, hence

$$
\boldsymbol{U}=\left(\begin{array}{cc}
1 & -u \\
u & 1
\end{array}\right), \quad \boldsymbol{V}=\left(\begin{array}{cc}
1 & v \\
-v & 1
\end{array}\right)
$$

Then $\boldsymbol{U}$ and $\boldsymbol{V}$ commute with $\gamma . \tilde{g}$ is real in particular if either of the following conditions is fulfilled.

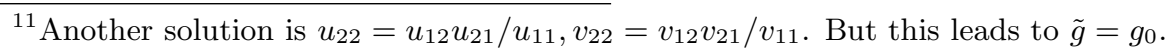


(1) $p, r$ real (which means $b, b^{\prime}$ real) and $u, v$ real.

(2) $\bar{r}=-\frac{1}{p}$ (which means $\bar{b}^{\prime}=b$ and $j=-j^{\prime} \in\{ \pm 1\}$ ) and $v=\bar{u}$.

The Ernst potential takes the form

$$
\mathcal{E}=\frac{(1+u v) \frac{p+r}{p-r}-\mathrm{i}(u-v) \frac{p r-1}{p r+1}+(u-\mathrm{i})(v-\mathrm{i})}{(1+u v) \frac{p+r}{p-r}-\mathrm{i}(u-v) \frac{p r-1}{p r+1}-(u-\mathrm{i})(v-\mathrm{i})} .
$$

By a shift of the origin of the coordinate $z$, we can arrange in both cases that

$$
\begin{array}{ll}
p=\rho^{-1}\left(z+b+j \mathfrak{r}_{+}\right), & r=\rho^{-1}\left(z-b+j^{\prime} \mathfrak{r}_{-}\right), \\
\mathfrak{r}_{ \pm}:=\sqrt{(z \pm b)^{2}+\rho^{2}}, & j, j^{\prime} \in\{ \pm 1\},
\end{array}
$$

where $b \in \mathbb{R}$ in case (1) and $b \in \mathrm{i} \mathbb{R}$ in case (2). Using

$$
\frac{p+r}{p-r}=\frac{1}{2 b}\left(j \mathfrak{r}_{+}+j^{\prime} \mathfrak{r}_{-}\right), \quad \frac{p r-1}{p r+1}=\frac{1}{2 b}\left(j \mathfrak{r}_{+}-j^{\prime} \mathfrak{r}_{-}\right),
$$

and introducing

$$
\mathfrak{a}=-b \frac{1+u v}{u-v}, \quad \mathfrak{l}=j b \frac{1-u v}{u-v}, \quad \mathfrak{m}=-j b \frac{u+v}{u-v},
$$

we obtain

$$
\mathcal{E}=\frac{\mathfrak{r}_{+}-j j^{\prime} \mathfrak{r}_{-}-\mathrm{i} \frac{\mathfrak{a}}{b}\left(\mathfrak{r}_{+}+j j^{\prime} \mathfrak{r}_{-}\right)-2(\mathfrak{m}+\mathrm{il})}{\mathfrak{r}_{+}-j j^{\prime} \mathfrak{r}_{-}-\mathrm{i} \frac{\mathfrak{a}}{b}\left(\mathfrak{r}_{+}+j j^{\prime} \mathfrak{r}_{-}\right)+2(\mathfrak{m}+\mathrm{il})} .
$$

Setting $j j^{\prime}=-1$, the cases (1) and (2) simply distinguish the non-extreme and the hyperextreme Kerr-NUT space-times (see e.g. [50]). The constants satisfy $\mathfrak{m}^{2}+\mathfrak{l}^{2}-\mathfrak{a}^{2}=b^{2}$.

Example 5.2. Let $n=2 N$. With the choices made in Remark 5.2, Proposition 5.1, part (1), implies that $\tilde{g}$, given by (4.8) with $g_{0}=I_{2}$, satisfies $(\gamma \tilde{g})^{2}=I_{2}$ and $\operatorname{tr}(\gamma \tilde{g})=0$. Choosing all parameters real, it follows that $\tilde{g}=I_{2}+\boldsymbol{U}(\tilde{\boldsymbol{R}} \tilde{\boldsymbol{X}})^{-1} \boldsymbol{V}$ determines a solution of the Ernst equation, provided that $\tilde{\boldsymbol{X}}$ is invertible ${ }^{12}$. For $N=1$, we are back to the preceding example. For $N=2$ let, for example,

$$
\begin{aligned}
\tilde{\boldsymbol{P}} & =\left(\begin{array}{cccc}
p & p \mathfrak{r}^{-1} & 0 & 0 \\
0 & p & 0 & 0 \\
0 & 0 & -p^{-1} & (p \mathfrak{r})^{-1} \\
0 & 0 & & -p^{-1}
\end{array}\right), \quad \tilde{\boldsymbol{R}}=\left(\begin{array}{cccc}
r_{1} & 0 & 0 & 0 \\
0 & r_{2} & 0 & 0 \\
0 & 0 & -r_{1}^{-1} & 0 \\
0 & 0 & 0 & -r_{2}^{-1}
\end{array}\right) \\
\boldsymbol{\Gamma} & =\left(\begin{array}{cccc}
0 & 0 & \mathrm{i} & 0 \\
0 & 0 & 0 & \mathrm{i} \\
-\mathrm{i} & 0 & 0 & 0 \\
0 & -\mathrm{i} & 0 & 0
\end{array}\right),
\end{aligned}
$$

where $p=\rho^{-1}(z+b+\mathfrak{r}), \mathfrak{r}= \pm \sqrt{(z+b)^{2}+\rho^{2}}$, and $r_{i}$ is also of the form (4.9) with a constant $b_{i}^{\prime} \neq b$. The conditions for $\boldsymbol{U}$ and $\boldsymbol{V}$ restrict these matrices to the form

$$
\boldsymbol{U}=\left(\begin{array}{cccc}
u_{1} & u_{3} & -u_{2} & -u_{4} \\
u_{2} & u_{4} & u_{1} & u_{3}
\end{array}\right), \quad \boldsymbol{V}=\left(\begin{array}{cc}
v_{1} & v_{2} \\
v_{3} & v_{4} \\
-v_{2} & v_{1} \\
-v_{4} & v_{3}
\end{array}\right)
$$

\footnotetext{
${ }^{12}$ The latter condition may indeed be violated, as shown in Remark 5.2.
} 
with constants $u_{i}, v_{i}$. If $r_{1}=r_{2}=: r$ (i.e. $b_{1}^{\prime}=b_{2}^{\prime}$ ), it turns out that $\tilde{g}$ does not depend on $r$ and $v_{i}$, and we obtain

$$
\mathcal{E}=\frac{\mathfrak{r}+\mathrm{i} \mathfrak{a}(z+b) \mathfrak{r}^{-1}-(\mathfrak{m}+\mathrm{il})}{\mathfrak{r}+\mathfrak{i} \mathfrak{a}(z+b) \mathfrak{r}^{-1}+(\mathfrak{m}+\mathrm{i} \mathfrak{l})}
$$

with the parameters

$$
\mathfrak{a}=\frac{u_{1}^{2}+u_{2}^{2}}{2\left(u_{1} u_{4}-u_{2} u_{3}\right)}, \quad \mathfrak{l}=\frac{u_{1}^{2}-u_{2}^{2}}{2\left(u_{1} u_{4}-u_{2} u_{3}\right)}, \quad \mathfrak{m}=-\frac{u_{1} u_{2}}{u_{1} u_{4}-u_{2} u_{3}},
$$

which satisfy $\mathfrak{m}^{2}-\mathfrak{a}^{2}+\mathfrak{l}^{2}=0$. $\mathcal{E}$ is the Ernst potential of an extreme Kerr-NUT space-time.

Example 5.3 (Multi-Kerr-NUT). According to Remark 5.1, there is a simple way to superpose solutions by block-diagonally composing their matrix data. Let

$$
\begin{array}{rlr}
\boldsymbol{U}_{i}=\left(\begin{array}{cc}
1 & -u_{i} \\
u_{i} & 1
\end{array}\right), & \boldsymbol{V}_{i}=\left(\begin{array}{cc}
1 & v_{i} \\
-v_{i} & 1
\end{array}\right) \\
\tilde{\boldsymbol{P}}_{i}=\left(\begin{array}{cc}
p_{i} & 0 \\
0 & -1 / p_{i}
\end{array}\right), & \tilde{\boldsymbol{R}}_{i}=\left(\begin{array}{cc}
r_{i} & 0 \\
0 & -1 / r_{i}
\end{array}\right),
\end{array}
$$

where $p_{i} \neq r_{k}, i, k=1, \ldots, N$, are given by (4.9), and either $b_{i}, b_{i}^{\prime}, u_{i}, v_{i} \in \mathbb{R}$ or $\bar{b}_{i}^{\prime}=b_{i} \in \mathbb{C}$, $j_{i}^{\prime}=-j_{i}, v_{i}=\bar{u}_{i} \in \mathbb{C}$ (cf. Example 5.1). Set

$$
\boldsymbol{U}=\left(\boldsymbol{U}_{1}, \ldots, \boldsymbol{U}_{N}\right), \quad \boldsymbol{V}=\left(\begin{array}{c}
\boldsymbol{V}_{1} \\
\vdots \\
\boldsymbol{V}_{N}
\end{array}\right)
$$

and $\tilde{\boldsymbol{P}}=\operatorname{block}-\operatorname{diag}\left(\tilde{\boldsymbol{P}}_{1}, \ldots, \tilde{\boldsymbol{P}}_{N}\right), \tilde{\boldsymbol{R}}=\operatorname{block}-\operatorname{diag}\left(\tilde{\boldsymbol{R}}_{1}, \ldots, \tilde{\boldsymbol{R}}_{N}\right), \boldsymbol{\Gamma}=\operatorname{block}-\operatorname{diag}(\gamma, \ldots, \gamma)$. With $g_{0}=I_{2}$, all assumptions of part (1) of Proposition 5.1 hold, hence with these data (4.8) determines a family of solutions of the Ernst equation. Obviously, such a solution is a superposition of $N$ (non-extreme, respectively hyperextreme) Kerr-NUT solutions ${ }^{13}$. More generally, in the same way we can superpose any number of solutions with matrix data of the form given in Example 5.2.

\subsection{Solutions of the Ernst equations in the Einstein-Maxwell case}

Choosing

$$
\gamma=\left(\begin{array}{ccc}
0 & \mathrm{i} & 0 \\
-\mathrm{i} & 0 & 0 \\
0 & 0 & -I_{m-2}
\end{array}\right)
$$

and writing

$$
v=\left(\begin{array}{c}
1 \\
\mathrm{i} \mathcal{E} \\
\sqrt{2} \Phi
\end{array}\right)
$$

with a complex function $\mathcal{E}$ and a complex $(m-2)$-component vector $\Phi,(5.2)$ takes the form

$$
\tilde{g}=\frac{2}{\mathcal{E}+\overline{\mathcal{E}}+2 \Phi^{\dagger} \Phi}\left(\begin{array}{ccc}
1 & \frac{\mathrm{i}}{2}\left(\mathcal{E}-\overline{\mathcal{E}}+2 \Phi^{\dagger} \Phi\right) & \sqrt{2} \Phi^{\dagger} \\
\frac{\mathrm{i}}{2}\left(\mathcal{E}-\overline{\mathcal{E}}-2 \Phi^{\dagger} \Phi\right) & \overline{\mathcal{E}} \mathcal{E} & \mathrm{i} \sqrt{2} \mathcal{E} \Phi^{\dagger} \\
\sqrt{2} \Phi & -\mathrm{i} \sqrt{2} \overline{\mathcal{E}} \Phi & 2 \Phi \Phi^{\dagger}-\frac{1}{2}\left(\mathcal{E}+\overline{\mathcal{E}}+2 \Phi^{\dagger} \Phi\right) I_{m-2}
\end{array}\right)
$$

\footnotetext{
${ }^{13}$ See e.g. $[6,24,51]$ for other derivations, and also [52], as well as the references cited there.
} 
(also see $[53,47])$. We have

$$
\mathcal{E}=\frac{1-\mathrm{i} \tilde{g}_{21}}{\tilde{g}_{11}}, \quad \Phi^{\top}=\frac{1}{\sqrt{2} \tilde{g}_{11}}\left(\tilde{g}_{31}, \ldots, \tilde{g}_{m-2,1}\right),
$$

where $^{\top}$ denotes transposition. In the following we consider the case $m=3$, where (4.2) becomes the system of Ernst equations

$$
\begin{aligned}
& (\operatorname{Re} \mathcal{E}+\bar{\Phi} \Phi)\left(\partial_{\rho}^{2}+\rho^{-1} \partial_{\rho}+\partial_{z}^{2}\right) \mathcal{E}=\left(\mathcal{E}_{\rho}\right)^{2}+\left(\mathcal{E}_{z}\right)^{2}+2 \bar{\Phi}\left[\Phi_{\rho} \mathcal{E}_{\rho}+\Phi_{z} \mathcal{E}_{z}\right], \\
& (\operatorname{Re} \mathcal{E}+\bar{\Phi} \Phi)\left(\partial_{\rho}^{2}+\rho^{-1} \partial_{\rho}+\partial_{z}^{2}\right) \Phi=\mathcal{E}_{\rho} \Phi_{\rho}+\mathcal{E}_{z} \Phi_{z}+2 \bar{\Phi}\left[\left(\Phi_{\rho}\right)^{2}+\left(\Phi_{z}\right)^{2}\right],
\end{aligned}
$$

which determine solutions of the stationary axially symmetric Einstein-Maxwell equations (without further matter fields). If $\mathcal{E}=1$ and $\Phi=0$, then $\tilde{g}$ reduces to

$$
g_{0}=\left(\begin{array}{ccc}
1 & 0 & 0 \\
0 & 1 & 0 \\
0 & 0 & -1
\end{array}\right)
$$

which corresponds to the Minkowski metric.

Example 5.4 (Demiański-Newman). Let $n=2$ and

$$
\boldsymbol{\Gamma}=\left(\begin{array}{cc}
0 & \mathrm{i} \\
-\mathrm{i} & 0
\end{array}\right), \quad \tilde{\boldsymbol{P}}=\left(\begin{array}{cc}
p & 0 \\
0 & -1 / p
\end{array}\right), \quad \tilde{\boldsymbol{R}}=\left(\begin{array}{cc}
r & 0 \\
0 & -1 / r
\end{array}\right),
$$

with $p, r$ as in (4.9) with constants $b, b^{\prime}$. Solving $g_{0} \gamma \boldsymbol{U}=\boldsymbol{U} \boldsymbol{\Gamma}$ and $\boldsymbol{\Gamma} \boldsymbol{V}=\boldsymbol{V} g_{0} \gamma$, and recalling that $\boldsymbol{U}$ and $\boldsymbol{V}$ enter the solution formula (4.8) only up to an overall factor, leads to

$$
\boldsymbol{U}=\left(\begin{array}{cc}
1 & -u \\
u & 1 \\
s & \mathrm{i} s
\end{array}\right), \quad \boldsymbol{V}=\left(\begin{array}{ccc}
1 & v & -t \\
-v & 1 & \mathrm{i} t
\end{array}\right) .
$$

According to Proposition 5.1, part (1), in order to obtain solutions of the Ernst equations it remains to determine conditions under which $\tilde{g}$ is Hermitian. By explicit evaluation one finds that this is so if one of the following sets of conditions is satisfied.

(1) $b, b^{\prime} \in \mathbb{R}$ and

$$
s t=-2 \frac{v+\mathrm{i}}{\bar{u}+\mathrm{i}} \operatorname{Im} u, \quad|v+\mathrm{i}|^{2} \operatorname{Im} u+|u-\mathrm{i}|^{2} \operatorname{Im} v=0, \quad 2 \operatorname{Im} u+|s|^{2}=0 .
$$

(2) $\bar{b}^{\prime}=b, j^{\prime}=-j, v=\bar{u}$ and $t=\bar{s}$.

Without restriction of generality we can set $b^{\prime}=-b$, so that $p$ and $r$ are given by (5.12). The Ernst potential $\mathcal{E}$ is again of the form (5.14), where now

$$
\mathfrak{a}=-b \frac{1+u v-s t}{u-v+\mathrm{i} s t}, \quad \mathfrak{l}=j b \frac{1-u v}{u-v+\mathrm{i} s t}, \quad \mathfrak{m}=-j b \frac{u+v}{u-v+\mathrm{i} s t} .
$$

The second Ernst potential is given by

$$
\Phi=\frac{2\left(q_{e}+\mathrm{i} q_{m}\right)}{\mathfrak{r}_{+}-j j^{\prime} \mathfrak{r}_{-}-\mathrm{i} \frac{\mathfrak{a}}{b}\left(\mathfrak{r}_{+}+j j^{\prime} \mathfrak{r}_{-}\right)+2(\mathfrak{m}+\mathrm{il})},
$$

where

$$
q_{e}=-\frac{j b}{\sqrt{2}} \frac{s(v-\mathrm{i})+t(u+\mathrm{i})}{u-v+\mathrm{i} s t}, \quad q_{m}=\mathrm{i} \frac{j b}{\sqrt{2}} \frac{s(v-\mathrm{i})-t(u+\mathrm{i})}{u-v+\mathrm{i} s t} .
$$


In both cases, the parameters $\mathfrak{a}, \mathfrak{l}, \mathfrak{m}, q_{e}, q_{m}$ are real and satisfy

$$
\mathfrak{m}^{2}-\mathfrak{a}^{2}+\mathfrak{l}^{2}-q_{e}^{2}-q_{m}^{2}=b^{2} .
$$

Cases (1) and (2) correspond to a non-extreme, respectively hyperextreme, Demiański-Newman space-time (see e.g. [50]). $q_{e}$ and $q_{m}$ are the electric and magnetic charge, respectively. Whereas (2) can be neatly expressed via (5.7), we have been unable so far to find a corresponding formulation for the conditions (1) in terms of the matrices $\tilde{\boldsymbol{P}}, \tilde{\boldsymbol{R}}, \boldsymbol{U}, \boldsymbol{V}$, also see Remark 5.4.

Example 5.5 (Harrison transformation). We can generate solutions of (5.18) via a Harrison transformation [48, 49]. A non-extreme Kerr-NUT solution (without charge) corresponds to the data

$$
\boldsymbol{U}_{0}=\left(\begin{array}{cc}
1 & -u_{0} \\
u_{0} & 1 \\
0 & 0
\end{array}\right), \quad \boldsymbol{V}_{0}=\left(\begin{array}{ccc}
1 & v_{0} & 0 \\
-v_{0} & 1 & 0
\end{array}\right)
$$

with real $u_{0}, v_{0}$. The matrix

$$
H=\frac{1}{1-|c|^{2}}\left(\begin{array}{ccc}
1 & \mathrm{i}|c|^{2} & \mathrm{i} \sqrt{2} c \\
-\mathrm{i}|c|^{2} & 1 & \sqrt{2} c \\
\mathrm{i} \sqrt{2} \bar{c} & -\sqrt{2} \bar{c} & -1-|c|^{2}
\end{array}\right)
$$

with $c \in \mathbb{C}$ satisfies (5.9) and (5.10). Then $\boldsymbol{U}^{\prime}=H \boldsymbol{U}_{0}$ and $\boldsymbol{V}^{\prime}=\boldsymbol{V}_{0} H$ satisfy $g_{0} \gamma \boldsymbol{U}^{\prime}=\boldsymbol{U}^{\prime} \boldsymbol{\Gamma}$ and $\boldsymbol{\Gamma} \boldsymbol{V}^{\prime}=\boldsymbol{V}^{\prime} g_{0} \gamma$, since $\boldsymbol{U}_{0}$ and $\boldsymbol{V}_{0}$ satisfy these conditions. Without effect on the solution of the chiral model, we can rescale these matrices to

$$
\begin{aligned}
& \boldsymbol{U}=\frac{1}{1+\mathrm{i} u_{0}|c|^{2}}\left(\begin{array}{cc}
1+\mathrm{i} u_{0}|c|^{2} & -\left(u_{0}-\mathrm{i}|c|^{2}\right) \\
u_{0}-\mathrm{i}|c|^{2} & 1+\mathrm{i} u_{0}|c|^{2} \\
\sqrt{2}\left(\mathrm{i}-u_{0}\right) \bar{c} & \mathrm{i} \sqrt{2}\left(\mathrm{i}-u_{0}\right) \bar{c}
\end{array}\right), \\
& \boldsymbol{V}=\frac{1}{1-\mathrm{i} v_{0}|c|^{2}}\left(\begin{array}{ccc}
1-\mathrm{i} v_{0}|c|^{2} & v_{0}+\mathrm{i}|c|^{2} & \sqrt{2}\left(\mathrm{i}+v_{0}\right) c \\
-\left(v_{0}+\mathrm{i}|c|^{2}\right) & 1-\mathrm{i} v_{0}|c|^{2} & -\mathrm{i} \sqrt{2}\left(\mathrm{i}+v_{0}\right) c
\end{array}\right),
\end{aligned}
$$

which have the form (5.17) and indeed satisfy (5.18). Using (5.13), the resulting Ernst potentials $\mathcal{E}$ and $\Phi$ can be written in the form (5.14), respectively (5.19), where now

$$
\mathfrak{a}=-b \frac{1+u_{0} v_{0}}{u_{0}-v_{0}}, \quad \mathfrak{l}=j b \frac{1-u_{0} v_{0}}{u_{0}-v_{0}} \frac{1+|c|^{2}}{1-|c|^{2}}, \quad \mathfrak{m}=-j b \frac{u_{0}+v_{0}}{u_{0}-v_{0}} \frac{1+|c|^{2}}{1-|c|^{2}}
$$

and

$$
\begin{aligned}
& q_{e}=\frac{2 j b\left[\left(u_{0} v_{0}-1\right) \operatorname{Re} c-\left(u_{0}+v_{0}\right) \operatorname{Im} c\right]}{\left(1-|c|^{2}\right)\left(u_{0}-v_{0}\right)}, \\
& q_{m}=-\frac{2 j b\left[\left(u_{0}+v_{0}\right) \operatorname{Re} c+\left(u_{0} v_{0}-1\right) \operatorname{Im} c\right]}{\left(1-|c|^{2}\right)\left(u_{0}-v_{0}\right)} .
\end{aligned}
$$

Example 5.6 (hyperextreme multi-Demiański-Newman). Let

$$
\boldsymbol{\Gamma}=\left(\begin{array}{ccccc}
0 & \mathrm{i} & & & \\
-\mathrm{i} & 0 & & & \\
& & \ddots & & \\
& & 0 & \mathrm{i} \\
& & & -\mathrm{i} & 0
\end{array}\right), \quad \begin{aligned}
& \tilde{\boldsymbol{P}}=\operatorname{block}-\operatorname{diag}\left(\tilde{\boldsymbol{P}}_{1}, \ldots, \tilde{\boldsymbol{P}}_{N}\right), \\
& \tilde{\boldsymbol{R}}=\operatorname{block}-\operatorname{diag}\left(\tilde{\boldsymbol{R}}_{1}, \ldots, \tilde{\boldsymbol{R}}_{N}\right), \quad \boldsymbol{V}=\left(\begin{array}{c}
\boldsymbol{V}_{1} \\
\vdots \\
\boldsymbol{V}_{N}
\end{array}\right), \\
&
\end{aligned}
$$


with

$$
\begin{array}{rlr}
\tilde{\boldsymbol{P}}_{i}=\left(\begin{array}{cc}
p_{i} & 0 \\
0 & -1 / p_{i}
\end{array}\right), & \tilde{\boldsymbol{R}}_{i}=\left(\begin{array}{cc}
r_{i} & 0 \\
0 & -1 / r_{i}
\end{array}\right), \\
\boldsymbol{U}_{i}=\left(\begin{array}{cc}
1 & -u_{i} \\
u_{i} & 1 \\
s_{i} & \mathrm{i} s_{i}
\end{array}\right), & \boldsymbol{V}_{i}=\left(\begin{array}{ccc}
1 & \bar{u}_{i} & -\bar{s}_{i} \\
-\bar{u}_{i} & 1 & \mathrm{i} \bar{s}_{i}
\end{array}\right),
\end{array}
$$

where $\bar{r}_{i}=-1 / p_{i}$. Then the conditions of Proposition 5.1 are obviously satisfied (with $\gamma$ and $g_{0}$ given by (5.15), respectively (5.16)). It follows that (4.8) determines a solution of the (EinsteinMaxwell-) Ernst equations. This is a superposition of $N$ hyperextreme Demiański-Newman solutions.

Remark 5.4. Whereas the hyperextreme multi-Demiański-Newman solutions are obtained in a straightforward way, this is not so in the non-extreme case. So far a suitable condition on the matrix data is lacking. Similar problems are known in other approaches, see e.g. [8, Section 34.8].

Example 5.7. Let $n=2 N$. With the choices made in Remark 5.2, Proposition 5.1, part (1), implies that the expression given by (4.8) with $g_{0}$ in (5.16) satisfies $(\gamma \tilde{g})^{2}=I_{3}$ and $\operatorname{tr}(\gamma \tilde{g})=1$. In order to obtain a solution of the Ernst equations, it suffices to arrange that $\tilde{g}$ is Hermitian. A sufficient condition is given by part (2) of Proposition 5.1. This leads to a huge family of solutions of the Einstein-Maxwell equations. The hyperextreme Demiański-Newman solution is just the simplest example in this family. Furthermore, such solutions can be superposed in the simple way described in Remark 5.1 and Example 5.6. An exploration of the corresponding space-times would be a difficult task.

\section{Conclusions}

We addressed the $m \times m$ non-autonomous chiral model in a new way, starting from a very simple and universal solution generating result within the bidifferential calculus approach. This resulted in an infinite family of exact solutions for any matrix size $m$, parametrized by matrices subject to a Sylvester equation. To solve the latter is a well-studied and fairly simple problem. At least in the compact form presented in this work, according to our knowledge these solutions have not appeared previously in the literature.

The non-autonomous chiral model originally appeared in reductions of Einstein's equations. We demonstrated in Section 5 that the "multi-solitons" on a flat background, known in the case of stationarity, axial symmetry and vacuum, respectively electrovacuum, are indeed contained in the family of solutions that we obtained in Section 4 for the non-autonomous chiral model equation. More precisely, we found conditions to be imposed on the matrices that parametrize the latter solutions such that (in the cases $m=2$, respectively $m=3$ ) they become solutions of the Ernst equation(s) of general relativity. Only in the case of non-extreme multi-DemiańskiNewman solutions we were not (yet) able to find a corresponding characterization of the matrix data.

Beyond the solutions found e.g. by Belinski and Zakharov, which in the present work correspond to diagonal matrices $\tilde{\boldsymbol{P}}$ and $\tilde{\boldsymbol{R}}$, there are solutions associated with non-diagonal matrix data. It may well be that such solutions can be obtained alternatively e.g. in the BelinskiZakharov framework with a dressing matrix involving higher order poles, or by taking suitable limits where some poles coincide. In any case, our approach yields these solutions directly. Moreover, relaxing the spectral condition for the matrices $\tilde{\boldsymbol{P}}$ and $\tilde{\boldsymbol{R}}$, the Sylvester equation has further solutions, provided that the matrix $\boldsymbol{V} \boldsymbol{U}$ on its right hand side is appropriately chosen. This is another possibility to obtain new solutions. Finally, we should mention the possibility to 
make sense of the limit ${ }^{14} n \rightarrow \infty$ (where $n \times n$ is the size of the matrices that parametrize the solutions). In conclusion, at present it is not quite clear what the generated class of solutions really embraces.

Moreover, using the original method of Belinski and Zakharov, in the Einstein-Maxwell case no appropriate reduction conditions could be found (cf. [54]), and a different approach had to be developed [23, 7]. We had less problems in this respect.

On the other hand, the Belinski-Zakharov approach, the modified approach of Alekseev [23] in the Einstein-Maxwell case, and others can also be used to generate "solitons" on a nonflat background. Perhaps a corresponding extension of Proposition 3.1 exists. This is also suggested by the relation with Darboux transformations in Appendix A. In any case, here we have a limitation of Proposition 3.1 (which is not a limitation of the bidifferential calculus framework, which offers various methods [1]), but we have the advantage of a very simple and general result that covers physically interesting cases.

The appearance of a Sylvester equation is a generic feature of the solution generating result formulated in Proposition 3.1 and in Appendix A (also see [1]). Sylvester equations and their simplest solutions, Cauchy-like matrices, frequently appeared in the integrable systems literature. But this is the first time we came across a Sylvester equation involving non-constant matrix data. A particularly nice feature is the fact that solutions can be superposed by simply composing their matrix data into bigger block-diagonal matrices. The corresponding Sylvester equation still has to be solved, but a unique solution exists if we impose a not very restrictive spectral condition on these matrix data.

In Appendix B we recovered two familiar Lax pairs for the non-autonomous chiral model from the general linear equation (2.7) in the bidifferential calculus framework. Our way toward exact solutions in Section 4 is more closely related to Maison's Lax pair than to that of Belinski and Zakharov. We eliminated the $\theta$-dependence, whereas in the Lax pair of Belinski and Zakharov the $\theta$-dependence is kept and it involves derivatives with respect to this "spectral parameter".

Our results extend beyond the Einstein-Maxwell case and are also applicable to higherdimensional gravity theories (see e.g. [9, 10, 11, 12, 13, 14, 15, 16, 17, 18, 19]). Besides that, other reductions of the non-autonomous chiral model (for some $m$ ) are of interest, see e.g. [20, 21], and the set of solutions that we obtained in this work will typically be reducible to solutions of them.

Since Proposition 3.1 actually generates solutions of a Miura transformation equation, we obtained simultaneously solutions of the Miura-dual of the non-autonomous chiral model equation: under the assumptions of Proposition 4.1, $\tilde{\phi}=\boldsymbol{U} \tilde{\boldsymbol{X}}^{-1} \boldsymbol{V}$ solves the equation (4.3). The further results in Section 4, in particular Example 4.1, then provide us with explicit families of solutions.

Proposition 3.1, respectively Theorem A.1, can actually be formulated and proved without explicit use of the two nonlinear equations involving only $\boldsymbol{P}$, respectively $\boldsymbol{R}$. In such a formulation, the theorem generates solutions of the nonlinear integrable equation (2.3), respectively (2.4), from solutions of linear equations. However, the equations for $\boldsymbol{P}$ and $\boldsymbol{R}$ arise as integrability conditions of the latter. In previous work [1,32, 33, 34], we chose $\boldsymbol{P}$ and $\boldsymbol{R}$ as d- and $\overline{\mathrm{d}}$-constant matrices, which indeed reduces the equations that have to be solved to only linear ones, and we recovered (and somewhat generalized) known soliton solution families. In case of the non-autonomous chiral model and, more specifically, its reduction to the Ernst equation, it turned out to be necessary to go beyond this level, and thus to consider genuine solutions of the nonlinear equations for $\boldsymbol{P}$ and $\boldsymbol{R}$, in order to obtain relevant solutions like those associated with multi-Kerr-NUT space-times and their (electrically and magnetically) charged generalizations. This also suggests a corresponding application of the theorem (or Proposition 3.1) to other integrable PDDEs.

\footnotetext{
${ }^{14}$ See e.g. $[55,56]$ for results on the operator Sylvester equation.
} 


\section{A Via a Darboux transformation and a projection to a non-iterative solution generating result}

Lemma A.1. Let $P$ be invertible. The transformation

$$
(\phi, g) \mapsto\left(\phi^{\prime}, g^{\prime}\right)=\left(\phi+X P X^{-1}, X P X^{-1} g\right),
$$

where $X$ is an invertible solution of (2.7) with $\mathbb{A}=\mathrm{d} \phi=(\overline{\mathrm{d}} g) g^{-1}$, and $\overline{\mathrm{d}} P=(\mathrm{d} P) P$, maps a solution of the Miura transformation equation (2.10) into another solution.

Proof. Using (2.7) and $\overline{\mathrm{d}} P=(\mathrm{d} P) P$, a direct computation leads to

$$
\left(\overline{\mathrm{d}} g^{\prime}\right) g^{\prime-1}-\mathrm{d} \phi^{\prime}=\mathbb{A}-\mathrm{d} \phi-X P X^{-1}\left[\mathbb{A}-(\overline{\mathrm{d}} g) g^{-1}\right] X P^{-1} X^{-1},
$$

which vanishes if $\mathbb{A}=\mathrm{d} \phi=(\overline{\mathrm{d}} g) g^{-1}$.

(A.1) is an essential part of a Darboux transformation, cf. [1]. In the following we will use this result to derive a theorem which essentially reduces to Proposition 3.1, see Remark A.2.

Lemma A.2. Let $(\boldsymbol{\phi}, \boldsymbol{g})$ be a solution of the Miura transformation equation (2.10) in $\operatorname{Mat}(n, n, \mathcal{B})$. Let $\boldsymbol{U} \in \operatorname{Mat}(m, n, \mathcal{B})$ and $\boldsymbol{V} \in \operatorname{Mat}(n, m, \mathcal{B})$ be $\mathrm{d}$ - and $\overline{\mathrm{d}}$-constant. If

$$
\phi=V \boldsymbol{U} \hat{\phi}
$$

with some $\hat{\phi} \in \operatorname{Mat}(n, n, \mathcal{B})$, then

$$
\phi=\boldsymbol{U} \hat{\boldsymbol{\phi}} \boldsymbol{V}, \quad g=\left(\boldsymbol{U g}^{-1} \boldsymbol{V}\right)^{-1}
$$

solve the Miura transformation equation (2.10) in $\operatorname{Mat}(m, m, \mathcal{B})$.

Proof. Since $(\boldsymbol{\phi}, \boldsymbol{g})$ is assumed to solve (2.10), we have

$$
\overline{\mathrm{d}} \boldsymbol{g}^{-1}=-\boldsymbol{g}^{-1} \mathrm{~d} \boldsymbol{\phi}=-\boldsymbol{g}^{-1} \boldsymbol{V} \boldsymbol{U} \mathrm{d} \hat{\boldsymbol{\phi}} .
$$

Multiplying by $\boldsymbol{U}$ from the left and by $\boldsymbol{V}$ from the right, we obtain

$$
\overline{\mathrm{d}} g^{-1}=-g^{-1} \mathrm{~d} \phi,
$$

which is equivalent to (2.10).

Theorem A.1. Let $(-\boldsymbol{R}, \boldsymbol{S})$ be a solution of the Miura transformation equation (2.10) in $\operatorname{Mat}(n, n, \mathcal{B})$, i.e.

$$
\overline{\mathrm{d}} \boldsymbol{S}=-(\mathrm{d} \boldsymbol{R}) \boldsymbol{S},
$$

and $\boldsymbol{S}$ invertible. Let $\boldsymbol{X}$ be an invertible solution of the linear equation $(2.7)$, now in $\operatorname{Mat}(n, n, \mathcal{B})$ and with invertible $\boldsymbol{P}$, hence

$$
\overline{\mathrm{d}} \boldsymbol{X}=(\mathrm{d} \boldsymbol{X}) \boldsymbol{P}-(\mathrm{d} \boldsymbol{R}) \boldsymbol{X}, \quad \overline{\mathrm{d}} \boldsymbol{P}=(\mathrm{d} \boldsymbol{P}) \boldsymbol{P} .
$$

In addition we require that

$$
\boldsymbol{X} \boldsymbol{P}-\boldsymbol{R} \boldsymbol{X}=\boldsymbol{V} \boldsymbol{Y}, \quad \overline{\mathrm{d}} \boldsymbol{R}=\boldsymbol{R} \mathrm{d} \boldsymbol{R}, \quad \overline{\mathrm{d}} \boldsymbol{Y}=(\mathrm{d} \boldsymbol{Y}) \boldsymbol{P},
$$

where $\boldsymbol{U} \in \operatorname{Mat}(m, n, \mathcal{B})$ and $\boldsymbol{V} \in \operatorname{Mat}(n, m, \mathcal{B})$ are $\mathrm{d}$ - and $\overline{\mathrm{d}}$-constant, and $\boldsymbol{Y} \in \operatorname{Mat}(n, n, \mathcal{B})$. Then also

$$
\phi=\boldsymbol{U} \boldsymbol{Y} \boldsymbol{X}^{-1} \boldsymbol{V} \quad \text { and } \quad g=\left(\boldsymbol{U} \boldsymbol{S}^{-1} \boldsymbol{X} \boldsymbol{P}^{-1} \boldsymbol{X}^{-1} \boldsymbol{V}\right)^{-1}
$$

solve the Miura transformation equation (2.10), and thus (2.3), respectively (2.4). 
Proof. Since we assume that $(-\boldsymbol{R}, \boldsymbol{S})$ solves the Miura transformation equation (2.10) in $\operatorname{Mat}(n, n, \mathcal{B})$, according to Lemma A.1 this also holds for the pair

$$
\phi=-\boldsymbol{R}+\boldsymbol{X} \boldsymbol{P} \boldsymbol{X}^{-1}, \quad \boldsymbol{g}=\boldsymbol{X} \boldsymbol{P} \boldsymbol{X}^{-1} \boldsymbol{S} .
$$

Using the first of (A.6), we find that (A.2) holds with $\hat{\boldsymbol{\phi}}=\boldsymbol{Y} \boldsymbol{X}^{-1}$. Now (A.3) yields the asserted formulas for $\phi$ and $g$. According to Lemma A.2, $\phi$ and $g$ solve the Miura transformation equation (2.10).

Together with (A.5), the first of (A.6) implies

$$
\boldsymbol{V} \boldsymbol{U}(\overline{\mathrm{d}} \boldsymbol{Y}-(\mathrm{d} \boldsymbol{Y}) \boldsymbol{P})=(\boldsymbol{R} \mathrm{d} \boldsymbol{R}-\overline{\mathrm{d}} \boldsymbol{R}) \boldsymbol{X},
$$

which is satisfied if the last two conditions of (A.6) hold.

Remark A.1. This theorem generalizes a previous result in [1], which has been applied in $[1,32,33,34]$ with d- and $\overline{\mathrm{d}}$-constant $\boldsymbol{P}, \boldsymbol{R}$, in which case only linear equations have to be solved in order to generate solutions of $(2.3)$, respectively (2.4).

The above derivation shows that the theorem may be regarded as a combination of a Darboux transformation (Lemma A.1), on the level of matrices of arbitrary size, and a projection mechanism (Lemma A.2). The projection idea can be traced back to work of Marchenko [57]. More generally, the above result can be formulated in terms of suitable operators, replacing the matrices that involve a size $n$.

The next remark shows that, with mild additional assumptions, Theorem A.1 reduces to Proposition 3.1.

Remark A.2. The transformation

$$
X \mapsto X Q, \quad Y \mapsto Y Q, \quad P \mapsto Q^{-1} P Q
$$

with any invertible $\boldsymbol{Q} \in \operatorname{Mat}(n, n, \mathcal{B})$, leaves the expressions for $\phi$ and $g$ in (A.7) invariant. This is then also a symmetry transformation of (A.5) and (A.6) if $\overline{\mathrm{d}} \boldsymbol{Q}^{-1}=\left(\mathrm{d} \boldsymbol{Q}^{-1}\right) \boldsymbol{P}$. As a consequence, under the assumptions that $\boldsymbol{Y}$ is invertible, without restriction of generality we can set $\boldsymbol{Y}=\boldsymbol{I}$, where $\boldsymbol{I}$ is the $n \times n$ identity matrix. Then $\phi$ is given by the expression in (3.2).

We further note that (A.4) and the second of (A.6) imply $\overline{\mathrm{d}}(\boldsymbol{R} \boldsymbol{S})=0$. Assuming that $\boldsymbol{R}$ is invertible, we thus have $\boldsymbol{S}=\boldsymbol{R}^{-1} \boldsymbol{C}$ with an invertible $\overline{\mathrm{d}}$-constant $\boldsymbol{C}$. The expression for $g$ in (A.7) now takes the form

$$
g=\left(\boldsymbol{U} \boldsymbol{C}^{-1} \boldsymbol{R} \boldsymbol{X}(\boldsymbol{X} \boldsymbol{P})^{-1} \boldsymbol{V}\right)^{-1}=\left(I-\boldsymbol{U}(\boldsymbol{X} \boldsymbol{P})^{-1} \boldsymbol{V}\right)^{-1}\left(\boldsymbol{U} \boldsymbol{C}^{-1} \boldsymbol{V}\right)^{-1},
$$

assuming temporarily invertibility of $\boldsymbol{U} \boldsymbol{C}^{-1} \boldsymbol{V}$. Together with $\phi$, this remains a solution of (2.10) if we drop the last factor, so that

$$
g=\left(I-\boldsymbol{U}(\boldsymbol{X P})^{-1} \boldsymbol{V}\right)^{-1}
$$

This expression also makes sense without the above additional invertibility assumptions. We can still translate it into a simpler form. From the first of (A.6), which now has the form of the last of (3.1), we obtain

$$
(\boldsymbol{R} \boldsymbol{X})^{-1}-(\boldsymbol{X} \boldsymbol{P})^{-1}=(\boldsymbol{R} \boldsymbol{X})^{-1} \boldsymbol{V U}(\boldsymbol{X} \boldsymbol{P})^{-1} .
$$

Multiplication by $\boldsymbol{U}$ from the left and by $\boldsymbol{V}$ from the right, and use in our last formula for $g$, leads to the expression for $g$ in (3.2). 


\section{B Linear systems for the non-autonomous chiral model}

\section{B.1 Maison's Lax pair}

Using the bidifferential calculus determined by (4.1), (2.7) with $\mathbb{A}=(\overline{\mathrm{d}} g) g^{-1}$ takes the form

$$
X_{\rho}+\rho^{-1} X_{\theta}=\left(g_{\rho}+\rho^{-1} g_{\theta}\right) g^{-1} X-X_{z} P e^{\theta}, \quad X_{z}=g_{z} g^{-1} X+\left(X_{\rho}-\rho^{-1} X_{\theta}\right) P e^{\theta},
$$

and (2.8) reads

$$
e^{-\theta}\left(P_{\rho}+\rho^{-1} P_{\theta}\right)=-P_{z} P, \quad P_{z}=e^{\theta}\left(P_{\rho}-\rho^{-1} P_{\theta}\right) P .
$$

Disregarding a constant solution (cf. Section B.2), we can eliminate the $\theta$-dependence in the latter equations via

$$
P=e^{-\theta} \tilde{P},
$$

with $\tilde{P}$ independent of $\theta$, and obtain

$$
\tilde{P}_{\rho}=\rho^{-1} \tilde{P}\left(I-\tilde{P}^{2}\right)\left(I+\tilde{P}^{2}\right)^{-1}, \quad \tilde{P}_{z}=2 \rho^{-1} \tilde{P}^{2}\left(I+\tilde{P}^{2}\right)^{-1} .
$$

Furthermore, setting

$$
X=e^{c_{1} \theta} \tilde{X}, \quad g=e^{c_{2} \theta} \tilde{g},
$$

with $\tilde{X}, \tilde{g}$ independent of $\theta$, the above linear system becomes

$$
\begin{aligned}
\tilde{X}_{\rho}\left(I+\tilde{P}^{2}\right) & =\left(\tilde{g}_{\rho}+c_{2} \rho^{-1} \tilde{g}\right) \tilde{g}^{-1} \tilde{X}-\tilde{g}_{z} \tilde{g}^{-1} \tilde{X} \tilde{P}-c_{1} \rho^{-1} \tilde{X}\left(I-\tilde{P}^{2}\right), \\
\tilde{X}_{z}\left(I+\tilde{P}^{2}\right) & =\tilde{g}_{z} \tilde{g}^{-1} \tilde{X}+\left(\tilde{g}_{\rho}+c_{2} \rho^{-1} \tilde{g}\right) \tilde{g}^{-1} \tilde{X} \tilde{P}-2 c_{1} \rho^{-1} \tilde{X} \tilde{P}
\end{aligned}
$$

Choosing

$$
\tilde{P}=p I
$$

with a function $p(\rho, z)$, the equations for $\tilde{P}$ can easily be integrated, which results in ${ }^{15}$

$$
p=\rho^{-1}\left(z+b \pm \sqrt{(z+b)^{2}+\rho^{2}}\right)
$$

where $b$ is an arbitrary constant. In terms of

$$
\hat{X}=\tilde{g}^{-1} \tilde{X}
$$

the above linear system, simplified by setting $c_{1}=c_{2}=0$, then takes the form

$$
\hat{X}_{\rho}=-\frac{p}{1+p^{2}}\left(\tilde{g}^{-1} \tilde{g}_{z}+p \tilde{g}^{-1} \tilde{g}_{\rho}\right) \hat{X}, \quad \hat{X}_{z}=\frac{p}{1+p^{2}}\left(\tilde{g}^{-1} \tilde{g}_{\rho}-p \tilde{g}^{-1} \tilde{g}_{z}\right) \hat{X}
$$

This system is equivalent to a linear system for the non-autonomous chiral model, first found by Maison in 1979 [4] (also see [41]).

\footnotetext{
${ }^{15}$ We note that $p$ is $\tilde{\boldsymbol{P}}_{1}$ in Section 4.2 .
} 


\section{B.2 The Belinski-Zakharov Lax pair}

Using instead of $\theta$ the variable

$$
\lambda=-\rho e^{\theta},
$$

(4.1) translates into

$$
\mathrm{d} f=-f_{z} \zeta_{1}-\rho^{-1} \lambda f_{\rho} \zeta_{2}, \quad \overline{\mathrm{d}} f=-\left(\rho \lambda^{-1} f_{\rho}+2 f_{\lambda}\right) \zeta_{1}+f_{z} \zeta_{2} .
$$

We consider the linear system (2.7) with $P=I$, which trivially solves (2.8), i.e.

$$
\overline{\mathrm{d}} X=\mathbb{A} X+\mathrm{d} X .
$$

Writing

$$
\mathbb{A}=-\frac{\rho}{\lambda} A \zeta_{1}+B \zeta_{2}
$$

the integrability condition (2.9) takes the form

$$
B_{\rho}-A_{z}=[A, B], \quad(\rho A)_{\rho}+(\rho B)_{z}=0,
$$

assuming that $A, B$ are $\lambda$-independent. Solving the first (zero curvature) condition by

$$
A=g_{\rho} g^{-1}, \quad B=g_{z} g^{-1},
$$

the second equation becomes the non-autonomous chiral model equation

$$
\left(\rho g_{\rho} g^{-1}\right)_{\rho}+\left(\rho g_{z} g^{-1}\right)_{z}=0
$$

The above linear equation leads to

$$
X_{\rho}=\frac{\rho \mathcal{U}+\lambda \mathcal{V}}{\rho^{2}+\lambda^{2}} X-\frac{2 \rho \lambda}{\rho^{2}+\lambda^{2}} X_{\lambda}, \quad X_{z}=\frac{\rho \mathcal{V}-\lambda \mathcal{U}}{\rho^{2}+\lambda^{2}} X+\frac{2 \lambda^{2}}{\rho^{2}+\lambda^{2}} X_{\lambda}
$$

where

$$
\mathcal{U}=\rho A, \quad \mathcal{V}=\rho B
$$

This is the Belinski-Zakharov Lax pair [6] (also see [7, Chapter 8]). We note that the "spectral parameter" $\lambda$ has its origin in a coordinate of the self-dual Yang-Mills equation. We also note that $\mathbb{A}=(\overline{\mathrm{d}} g) g^{-1}$ (using $\left.g_{\lambda}=0\right)$.

\section{Some proofs}

\section{C.1 Proof of Lemma 4.1}

(1) Assuming that $\boldsymbol{I}+\tilde{\boldsymbol{P}}^{2}$ is invertible, the system (4.4) can be decoupled into

$$
\tilde{\boldsymbol{P}}_{\rho}=\rho^{-1} \tilde{\boldsymbol{P}}\left(\boldsymbol{I}-\tilde{\boldsymbol{P}}^{2}\right)\left(\boldsymbol{I}+\tilde{\boldsymbol{P}}^{2}\right)^{-1}, \quad \tilde{\boldsymbol{P}}_{z}=2 \rho^{-1} \tilde{\boldsymbol{P}}^{2}\left(\boldsymbol{I}+\tilde{\boldsymbol{P}}^{2}\right)^{-1},
$$

which can also be written as

$$
\left(\tilde{\boldsymbol{P}}^{-1}\right)_{\rho}=-\rho^{-1} \tilde{\boldsymbol{P}}^{-1}\left(\boldsymbol{I}-\tilde{\boldsymbol{P}}^{2}\right)\left(\boldsymbol{I}+\tilde{\boldsymbol{P}}^{2}\right)^{-1}, \quad\left(\tilde{\boldsymbol{P}}^{-1}\right)_{z}=-2 \rho^{-1}\left(\boldsymbol{I}+\tilde{\boldsymbol{P}}^{2}\right)^{-1},
$$


assuming that $\tilde{\boldsymbol{P}}$ is invertible. Subtraction yields

$$
\left(\tilde{\boldsymbol{P}}-\tilde{\boldsymbol{P}}^{-1}\right)_{\rho}=-\rho^{-1}\left(\tilde{\boldsymbol{P}}-\tilde{\boldsymbol{P}}^{-1}\right), \quad\left(\tilde{\boldsymbol{P}}-\tilde{\boldsymbol{P}}^{-1}\right)_{z}=2 \rho^{-1} \boldsymbol{I},
$$

which can be integrated to

$$
\tilde{\boldsymbol{P}}-\tilde{\boldsymbol{P}}^{-1}=2 \rho^{-1}(z \boldsymbol{I}+\boldsymbol{B}),
$$

with a constant matrix $\boldsymbol{B}$. This implies (4.5).

(2) Let $\tilde{\boldsymbol{P}}$ satisfy (4.5) with a constant matrix $\boldsymbol{B}$. Then $\tilde{\boldsymbol{P}}$ is invertible, since the existence of a non-vanishing vector annihilated by $\tilde{\boldsymbol{P}}$ would be in conflict with (4.5). Thus (C.2) holds, which implies $[\tilde{\boldsymbol{P}}, \boldsymbol{B}]=0$. Differentiation of (4.5) with respect to $\rho$, and elimination of $z \boldsymbol{I}+\boldsymbol{B}$ with the help of (4.5) or equivalently (C.2), leads to

$$
0=\tilde{\boldsymbol{P}}_{\rho} \tilde{\boldsymbol{P}}+\tilde{\boldsymbol{P}} \tilde{\boldsymbol{P}}_{\rho}+2 \rho^{-2}(z \boldsymbol{I}+\boldsymbol{B}) \tilde{\boldsymbol{P}}-2 \rho^{-1}(z \boldsymbol{I}+\boldsymbol{B}) \tilde{\boldsymbol{P}}_{\rho}=\left(\tilde{\boldsymbol{P}}+\tilde{\boldsymbol{P}}^{-1}\right) \tilde{\boldsymbol{P}}_{\rho}+\rho^{-1}\left(\tilde{\boldsymbol{P}}^{2}-\boldsymbol{I}\right),
$$

where we used the assumption $\left[\tilde{\boldsymbol{P}}_{\rho}, \tilde{\boldsymbol{P}}\right]=0$. If $\boldsymbol{I}+\tilde{\boldsymbol{P}}^{2}$ is invertible, the resulting equation is the first of (C.1). In the same way we obtain the second of (C.1). (C.1) is equivalent to (4.4).

\section{C.2 Proof of Proposition 5.1}

Using (4.7), (5.4) and (5.5), we find

$$
\tilde{\boldsymbol{R}}(\tilde{\boldsymbol{X}} \tilde{\boldsymbol{P}}+\boldsymbol{\Gamma} \tilde{\boldsymbol{R}} \tilde{\boldsymbol{X}} \boldsymbol{\Gamma})-(\tilde{\boldsymbol{X}} \tilde{\boldsymbol{P}}+\boldsymbol{\Gamma} \tilde{\boldsymbol{R}} \tilde{\boldsymbol{X}} \boldsymbol{\Gamma}) \tilde{\boldsymbol{P}}=0,
$$

so that the spectrum condition implies

$$
\boldsymbol{\Gamma} \tilde{\boldsymbol{R}} \tilde{\boldsymbol{X}} \boldsymbol{\Gamma}=-\tilde{\boldsymbol{X}} \tilde{\boldsymbol{P}}
$$

With the help of this result we obtain

$$
\begin{aligned}
g_{0} \gamma\left(I+\boldsymbol{U}(\tilde{\boldsymbol{R}} \tilde{\boldsymbol{X}})^{-1} \boldsymbol{V}\right) & =g_{0} \gamma+\boldsymbol{U} \boldsymbol{\Gamma}(\tilde{\boldsymbol{R}} \tilde{\boldsymbol{X}})^{-1} \boldsymbol{V}=g_{0} \gamma-\boldsymbol{U}(\boldsymbol{\Gamma} \tilde{\boldsymbol{X}} \tilde{\boldsymbol{P}})^{-1} \boldsymbol{V} \\
& =\left(I-\boldsymbol{U}(\tilde{\boldsymbol{X}} \tilde{\boldsymbol{P}})^{-1} \boldsymbol{V}\right) g_{0} \gamma .
\end{aligned}
$$

Using $\left(g_{0} \gamma\right)^{2}=I$, the condition $(\gamma \tilde{g})^{2}=I$ for $(4.8)$ is therefore equivalent to

$$
\left(I-\boldsymbol{U}(\tilde{\boldsymbol{X}} \tilde{\boldsymbol{P}})^{-1} \boldsymbol{V}\right)\left(I+\boldsymbol{U}(\tilde{\boldsymbol{R}} \tilde{\boldsymbol{X}})^{-1} \boldsymbol{V}\right)=I .
$$

Expanding the left hand side and using the Sylvester equation (4.7) to eliminate $\boldsymbol{V} \boldsymbol{U}$, this indeed turns out to be satisfied. To complete the proof of (1), it remains to derive the trace formula. Using (4.8), (4.7) and (C.3), we obtain

$$
\begin{aligned}
\operatorname{tr}(\gamma \tilde{g})-\operatorname{tr}\left(\gamma g_{0}\right) & =\operatorname{tr}\left((\tilde{\boldsymbol{R}} \tilde{\boldsymbol{X}})^{-1} \boldsymbol{V} g_{0} \gamma \boldsymbol{U}\right)=\operatorname{tr}\left((\tilde{\boldsymbol{R}} \tilde{\boldsymbol{X}})^{-1} \boldsymbol{V} \boldsymbol{U} \boldsymbol{\Gamma}\right) \\
& =\operatorname{tr}\left((\tilde{\boldsymbol{R}} \tilde{\boldsymbol{X}})^{-1}(\tilde{\boldsymbol{X}} \tilde{\boldsymbol{P}}-\tilde{\boldsymbol{R}} \tilde{\boldsymbol{X}}) \boldsymbol{\Gamma}\right)=-\operatorname{tr}(\boldsymbol{\Gamma})+\operatorname{tr}\left((\tilde{\boldsymbol{R}} \tilde{\boldsymbol{X}})^{-1} \tilde{\boldsymbol{X}} \tilde{\boldsymbol{P}} \boldsymbol{\Gamma}\right) \\
& =-\operatorname{tr}(\boldsymbol{\Gamma})-\operatorname{tr}\left((\tilde{\boldsymbol{R}} \tilde{\boldsymbol{X}})^{-1} \boldsymbol{\Gamma} \tilde{\boldsymbol{R}} \tilde{\boldsymbol{X}}\right)=-2 \operatorname{tr}(\boldsymbol{\Gamma}) .
\end{aligned}
$$

In order to prove (2), we consider the Hermitian conjugate of the Sylvester equation (4.7). By use of (5.7), and with the help of $g_{0} \gamma \boldsymbol{U}=\boldsymbol{U} \boldsymbol{\Gamma}, \boldsymbol{\Gamma} \boldsymbol{V}=\boldsymbol{V} g_{0} \gamma$ and $\left(g_{0} \gamma\right)^{2}=I$, it takes the form

$$
\boldsymbol{V} \boldsymbol{U}=-\left(\boldsymbol{\Gamma} \tilde{\boldsymbol{X}}^{\dagger} \boldsymbol{\Gamma}\right) \tilde{\boldsymbol{P}}+\tilde{\boldsymbol{R}}\left(\boldsymbol{\Gamma} \tilde{\boldsymbol{X}}^{\dagger} \boldsymbol{\Gamma}\right)
$$

By comparison with the original Sylvester equation, the spectrum condition allows us to conclude that

$$
\tilde{\boldsymbol{X}}^{\dagger}=-\boldsymbol{\Gamma} \tilde{\boldsymbol{X}} \boldsymbol{\Gamma}
$$

Together with (C.3) this implies

$$
(\tilde{\boldsymbol{R}} \tilde{\boldsymbol{X}})^{\dagger}=\tilde{\boldsymbol{X}}^{\dagger} \tilde{\boldsymbol{R}}^{\dagger}=-\boldsymbol{\Gamma} \tilde{\boldsymbol{X}} \boldsymbol{\Gamma}^{2} \tilde{\boldsymbol{P}} \boldsymbol{\Gamma}=-\boldsymbol{\Gamma} \tilde{\boldsymbol{X}} \tilde{\boldsymbol{P}} \boldsymbol{\Gamma}=\tilde{\boldsymbol{R}} \tilde{\boldsymbol{X}}
$$

It follows that $\boldsymbol{U}(\tilde{\boldsymbol{R}} \tilde{\boldsymbol{X}})^{-1} \boldsymbol{V} g_{0}$ is Hermitian, and thus also $\tilde{g}$ given by (4.8). 


\section{Acknowledgements}

We would like to thank Vladimir S. Manko and anonymous referees for helpful comments. During the course of this work, N.K. has been at the Max-Planck-Institute for Dynamics and Self-Organization in Göttingen.

\section{References}

[1] Dimakis A., Müller-Hoissen F., Bidifferential graded algebras and integrable systems, Discrete Contin. Dyn. Syst. 2009 (2009), suppl., 208-219, arXiv:0805.4553.

[2] Kinnersley W., Symmetries of the stationary Einstein-Maxwell field equations. I, J. Math. Phys. 18 (1977), 1529-1537.

[3] Maison D., Are the stationary, axially symmetric Einstein equations completely integrable?, Phys. Rev. Lett. 41 (1978), 521-522.

[4] Maison D., On the complete integrability of the stationary, axially symmetric Einstein equations, J. Math. Phys. 20 (1979), 871-877.

[5] Belinskiǐ V., Zakharov V., Integration of the Einstein equations by means of the inverse scattering problem technique and construction of exact soliton solutions, Soviet Phys. JETP 48 (1978), 985-994.

[6] Belinskiǐ V., Sakharov V., Stationary gravitational solitons with axial symmetry, Soviet Phys. JETP 50 (1979), 1-9.

[7] Belinski V., Verdaguer E., Gravitational solitons, Cambridge Monographs on Mathematical Physics, Cambridge University Press, Cambridge, 2001.

[8] Stephani H., Kramer D., MacCallum M., Hoenselaers C., Herlt E., Exact solutions of Einstein's field equations, Cambridge Monographs on Mathematical Physics, Cambridge University Press, Cambridge, 2003.

[9] Lee S.-C., Generalized Neugebauer-Kramer transformations for nonlinear sigma models, Phys. Lett. B 164 (1985), 75-79.

[10] Lee S.-C., Soliton transformations for axially symmetric higher-dimensional gravity. II. Belinkii-Zakharov $N$-soliton transformations, J. Math. Phys. 28 (1987), 901-904.

[11] Wei Y.-H., Extended Ernst equation and five-dimensional axisymmetric Kaluza-Klein solutions, Classical Quantum Gravity 18 (2001), 2163-2170.

[12] Harmark T., Stationary and axisymmetric solutions of higher-dimensional general relativity, Phys. Rev. D 70 (2004), 124002, 25 pages, hep-th/0408141.

[13] Pomeransky A.A., Complete integrability of higher-dimensional Einstein equations with additional symmetry and rotating black holes, Phys. Rev. D 73 (2006), 044004, 5 pages, hep-th/0507250.

[14] Azuma T., Koikawa T., An infinite number of stationary soliton solutions to the five-dimensional vacuum Einstein equation, Progr. Theoret. Phys. 116 (2006), 319-328, hep-th/0512350.

[15] Iguchi H., Mishima T., Solitonic generation of vacuum solutions in five-dimensional general relativity, Phys. Rev. D 74 (2006), 024029, 17 pages, hep-th/0605090.

[16] Tomizawa S., Morisawa Y., Yasui Y., Vacuum solutions of five dimensional Einstein equations generated by inverse scattering method, Phys. Rev. D 73 (2006), 064009, 8 pages, hep-th/0512252.

[17] Yazadjiev S.S., 5D Einstein-Maxwell solitons and concentric rotating dipole black rings, Phys. Rev. D 78 (2008), 064032, 11 pages, arXiv:0805.1600.

[18] Emparan R., Reall H.S., Black holes in higher dimensions, Living Rev. Relativity 11 (2008), 6, 87 pages, arXiv:0801.3471.

[19] Figueras P., Jamsin E., Rocha J.V., Virmani A., Integrability of five-dimensional minimal supergravity and charged rotating black holes, Classical Quantum Gravity 27 (2010), 135011, 37 pages, arXiv:0912.3199.

[20] Mikhailov A.V., Yaremchuk A.I., Cylindrically symmetric solutions of the non-linear chiral field model $(\sigma$ model), Nuclear Phys. B 202 (1982), 508-522.

[21] Gutshabash E.Sh., Lipovskii V.D., Exact solutions of a nonlinear sigma-model in a curved space and the theory of magnetically ordered media with variable saturation magnetization, J. Math. Sci. 77 (1995), 3063-3068.

[22] Alekseev G.A., N-soliton solutions of Einstein-Maxwell equations, JETP Lett. 32 (1980), 277-279. 
[23] Alekseev G.A., Exact solutions in the general theory of relativity, Proc. Steklov Inst. Math. 176 (1988), 215-262.

[24] Neugebauer G., A general integral of the axially symmetric Einstein equations, J. Phys. A: Math. Gen. 13 (1980), L19-L21.

[25] Neugebauer G., Kramer D., Einstein-Maxwell solitons, J. Phys. A: Math. Gen. 16 (1983), $1927-1936$.

[26] Kramer D., Neugebauer G., Matos T., Bäcklund transforms of chiral fields, J. Math. Phys. 32 (1991), $2727-2730$.

[27] Korotkin D., Finite-gap solutions of the stationary axisymmetric Einstein equation in vacuum, Theoret. and Math. Phys. 77 (1989), 1018-1031.

[28] Manko V., Martín J., Ruiz E., Extended family of the electrovac two-solitons for the Einstein-Maxwell equations, Phys. Rev. D 51 (1995), 4187-4191.

[29] Ruiz E., Manko V., Martín J., Extended N-soliton solution of the Einstein-Maxwell equations, Phys. Rev. D 51 (1995), 4192-4197.

[30] Masuda T., Sasa N., Fukuyama T., Neugebauer-Kramer solutions of the Ernst equation in Hirota's direct method, J. Phys. A: Math. Gen. 31 (1998), 5717-5731.

[31] Burtsev S.P., Zakharov V.E., Mikhailov A.V., Inverse scattering method with variable spectral parameter, Theoret. and Math. Phys. 70 (1987), 227-240.

[32] Dimakis A., Müller-Hoissen F., Solutions of matrix NLS systems and their discretizations: a unified treatment, Inverse Problems 26 (2010), 095007, 55 pages.

[33] Dimakis A., Müller-Hoissen F., Bidifferential calculus approach to AKNS hierarchies and their solutions, SIGMA 6 (2010), 055, 27 pages, arXiv:1004.1627.

[34] Dimakis A., Kanning N., Müller-Hoissen F., Bidifferential calculus, matrix SIT and sine-Gordon equations, Acta Polytechnica 51 (2011), 33-37, arXiv:1011.1737.

[35] Witten L., Static axially symmetric solutions of self-dual SU(2) gauge fields in Euclidean four-dimensional space, Phys. Rev. D 19 (1979), 718-720.

[36] Forgács P., Horváth Z., Palla L., Generating the Bogomolny-Prasad-Sommerfield one-monopole solution by a Bäcklund transformation, Phys. Rev. Lett. 45 (1980), 505-508.

[37] Bais F.A., Sasaki R., On the complete integrability of the static axially symmetric self-dual gauge field equations for an arbitrary group, Nuclear Phys. B 195 (1982), 522-540.

[38] Ward R.S., Stationary axisymmetric space-times: a new approach, Gen. Relativity Gravitation 15 (1983), 105-109.

[39] Mason L.J., Woodhouse N.M.J., Integrability, self-duality, and twistor theory, London Mathematical Society Monographs. New Series, Vol. 15, Oxford Science Publications, The Clarendon Press, Oxford University Press, New York, 1996.

[40] Klein C., Richter O., Ernst equation and Riemann surfaces. Analytical and numerical methods, Lecture Notes in Physics, Vol. 685, Springer-Verlag, Berlin, 2005.

[41] Kanning N., Integrable Systeme in der Allgemeinen Relativitätstheorie: ein Bidifferentialkalkül-Zugang, Diploma Thesis, University of Göttingen, 2010.

[42] Higham N.J., Computing real square roots of a real matrix, Linear Algebra Appl. 88/89 (1987), 405-430.

[43] Bernstein D.S., Matrix mathematics. Theory, facts, and formulas, 2nd ed., Princeton University Press, Princeton, NJ, 2009.

[44] Zakharov V.E., Mikhailov A.V., Relativistically invariant two-dimensional models of field theory which are integrable by means of the inverse scattering problem method, Soviet Phys. JETP 47 (1978), 1017-1027.

[45] Eichenherr H., Forger M., More about non-linear sigma models on symmetric spaces, Nuclear Phys. B 164 (1980), 528-535.

[46] Mazur P.O., Proof of uniqueness of the Kerr-Newman black hole solution, J. Phys. A: Math. Gen. 15 (1982), 3173-3180.

[47] Gürses M., Inverse scattering, differential geometry, Einstein-Maxwell solitons and one soliton Bäcklund transformations, in Solutions of Einstein's Equations: Techniques and Results (Retzbach, 1983), Editors C. Hoenselaers and W. Dietz, Lecture Notes in Phys., Vol. 205, Springer, Berlin, 1984, 199-234.

[48] Harrison B.K., New solutions of the Einstein-Maxwell equations from old, J. Math. Phys. 9 (1968), 17441752 . 
[49] Kinnersley W., Generation of stationary Einstein-Maxwell fields, J. Math. Phys. 14 (1973), 651-653.

[50] Aguilar-Sánchez J., García A.A., Manko V.S., Demiański-Newman solution revisited, Gravit. Cosmol. 7 (2001), 149-152, gr-qc/0106011.

[51] Kramer D., Neugebauer G., The superposition of two Kerr solutions, Phys. Lett. A 75 (1980), $259-261$.

[52] Neugebauer G., Hennig J., Non-existence of stationary two-black-hole configurations, Gen. Relativity Gravitation 41 (2009), 2113-2130, arXiv:0905.4179.

[53] Gürses M., Xanthopoulos B., Axially symmetric, static self-dual SU(3) gauge fields and stationary EinsteinMaxwell metrics, Phys. Rev. D 26 (1982), 1912-1915.

[54] Alekseev G., Thirty years of studies of integrable reductions of Einstein's field equations, arXiv:1011.3846.

[55] Bhatia R., Rosenthal P., How and why to solve the operator equation $A X-X B=Y$, Bull. London Math. Soc. 29 (1997), 1-21.

[56] Garimella R.V., Hrynkiv V., Sourour A.R., A solution of an operator equation related to the KdV equation, Linear Algebra Appl. 418 (2006), 788-792.

[57] Marchenko V., Nonlinear equations and operator algebras, Mathematics and its Applications, Vol. 17, D. Reidel Publishing Co., Dordrecht, 1988. 